EUROPEAN LABORATORY FOR PARTICLE PHYSICS

CERN-EP/98-139

18 September 1998

\title{
HIGH RATE BEHAVIOR AND DISCHARGE LIMITS IN MICRO-PATTERN DETECTORS
}

\author{
A. Bressan, M. Hoch, P. Pagano, L. Ropelewski and F. Sauli \\ (CERN, Geneva, Switzerland) \\ S. Biagi \\ (Univ. Liverpool) \\ A. Buzulutskov \\ (Budker Institute for Nuclear Physics, Novosibirsk, Russia) \\ M. Gruwé \\ (DESY-Univ. Hamburg, Germany) \\ G. De Lentdecker \\ (ULB Bruxelles, Belgium) \\ D. Moermann \\ (Univ. Karlsruhe, Germany) \\ A. Sharma \\ (GSI Darmsdtadt, Germany)
}

\begin{abstract}
We present and discuss a set of systematic measurements, carried out with gaseous proportional micro-pattern detectors, in order to assess their maximum gain when irradiated with high-rate soft X-rays and heavily ionizing alpha particles. The inventory of detectors tested includes: microstrips, micromegas, micro-dot, gas electron multiplier, CAT (compteur à trous), trench (or groove), micro-CAT (or WELL) detectors, as well as systems with two elements of gaseous amplification in cascade. We confirm the general trend of all single-stage detectors to follow Raether's criterion, i.e. a spontaneous transition from avalanche to streamer, followed by a discharge, when the avalanche size reaches a value of a few $10^{7}$; a noticeable exception is the micro-dot counter holding more than $10^{8}$. In multiple structures, where the gain is shared between two devices in cascade, the maximum overall gain under irradiation is increased by at least one order of magnitude; we speculate this to be a consequence of a voltage dependence of Raether's limit, larger for low operating potentials. Our conclusion is that only multiple devices can guarantee a sufficient margin of reliability for operation in harsh LHC running conditions.
\end{abstract}

Submitted to Nuclear Instruments and Methods in Physics Research

Corresponding author:

fabio.sauli@cern.ch 



\section{INTRODUCTION}

Triggered by the invention, ten years ago, of the micro-strip gas chamber (MSGC) [1], during the last decade a new generation of fast, performing gas detectors has emerged, relying for manufacturing on more or less sophisticated photo-lithographic patterning technologies. A nonexhaustive list of such innovative devices includes the micro-gap [2], micro-dot [3], "compteur à trous" (CAT) [4], small gap [5], micromegas [6], gas electron multiplier (GEM) [7], micro-CAT [8], micro-groove and WELL detectors [9, 10]. For a recent review see for example [11, 12]. All these devices, here collectively named micro-pattern detectors, share a common characteristics setting them aside in behavior from multiwire counters: a high electric field extends over a large fraction or all of the gap between anodes and cathodes, generally very narrow (few tens to few hundred $\mu \mathrm{m}$ ), and in most cases high field singularities also exist close to the cathode surface.

The presence of a high field on cathode edges limits the maximum voltage that can be reached in MSGCs, even in absence of radiation, due to spontaneous field emission [13]. It has been observed that under high irradiation rate and/or exposure to heavily ionizing tracks, occasional transitions from proportional avalanche to streamer occur at high operating voltage, most of the times followed by a discharge, harmful and often fatal to the delicate structures and readout electronics. Proximity of the electrodes to an insulating or slightly conducting support, with the ensuing surface field enhancement and dynamic charging-up, appears to aggravate the situation $[14,15]$.

Early observations of a reduction in the maximum operating voltage for MSGC exposed to heavily ionizing tracks (5.6 MeV $\alpha$ particles from ${ }^{241} \mathrm{Am}$ ) were reported by the authors of Ref. [16]. Probably due to the low rate and the limited size of the irradiated area, the reduction was moderate and appeared compatible with safe operation at the gains needed for detection of minimum ionizing particles $\left(2-310^{3}\right)$. A near catastrophic experience was however reported by the Heidelberg group testing large size prototype MSGCs in a mixed field beam, containing heavily ionizing nuclear fragments: many of the detectors did not survive the exposure, suffering irreversible damages attributed to discharges [17]. A similar, albeit less dramatic, negative experience was reported by the authors of Ref. [18], who observed a continuous deterioration of performance and a decrease of operating voltage during several months of exposure to a high intensity beam. These, and other observations, have stimulated detailed studies of discharge mechanisms in micro-pattern detectors under controlled laboratory conditions [19-22], as well as encouraged the search for structures sturdier than the fragile standard MSGCs [23]. At the same time, it encouraged the development of innovative devices possibly capable of a more reliable operation.

This paper describes systematic measurements carried out on a representative inventory of recently introduced micro-pattern detectors, exposed to radiation as close as possible to the real operating conditions. 


\section{TRANSITION FROM AVALANCHE TO STREAMER}

The complex physical processes leading to the transition from a proportional avalanche multiplication to a streamer, and to the ensuing discharge, have been a classic subject of study and are discussed in many textbooks [24-26]. A mathematical model describing the avalanche development and the transition from proportional avalanche to streamer has been developed for the particular case of a uniform electric field [27]. More recently, the subject has been analyzed in parallel plate chambers, both experimentally and theoretically [28, 29]. The major outcomes of the studies can be summarized as follows. While at low gas pressures secondary feedback mechanisms involving photons and ions are significant (and can lead to the so-called slow breakdown), at pressures close to atmospheric the dominant mechanism of discharge is a fast, photon-mediated transition from proportional multiplication to streamer, followed by breakdown. Detailed simulations of the time development of the avalanche and of the streamer have been performed; the results match qualitatively rather well the observations. The general conclusion is that when the total charge in the avalanche exceeds a value in the range between $10^{7}$ and $10^{8}$ electron-ion pairs, the so-called Raether's limit, the enhancement of the electric field in front and behind the primary avalanche is such as to induce the fast growth of secondary avalanches, and the appearance of a long, filament-like forward and backward charge propagation named streamer. In a uniform, strong electric field, the streamer propagates all the way through the gap. The outcome of the process is the creation of a densely ionized, low-resistivity channel between anode and cathode, inevitably leading to discharge; the onset of the process is very fast, few tens of ns, hence the name of fast breakdown. The observed early failure induced by heavily ionizing tracks is then the obvious consequence of the large avalanche size reached with even moderate multiplication factors. It was obscure however, and it is still rather unclear today, why a high flux of low specific ionization radiation (such as soft $\mathrm{X}$-rays) induces a similar transition.

In a typical thin-gap gas counter, exposure to particles from an external source of heavily ionizing radiation (such as $\alpha$ particles) results in the deposition of $\sim 10^{4}$ pairs per $\mathrm{cm}$; one would expect the Raether limit to be reached at gains between $10^{3}$ and $10^{4}$, as indeed observed. This is within the range of gains required to gas devices in order to detect minimum ionizing particles, and is a direct outcome of the signal to noise features offered by modern highly integrated fast electronics, combined with the high rate requirements demanding a narrow sensitive gap that can be quickly cleared from the charge produced in the avalanches. The margin between the voltage required for efficient detection of relativistic particles, and the appearance of discharges in the presence of a high flux of heavily ionizing background is therefore small, if any, and depends critically on the choice of the operating parameters, as well as on the composition of the radiation field. This has motivated the resurgence of detailed studies on the subject by many groups developing modern fast micro-pattern detectors. 
It should be recalled that in multiwire proportional chambers and derivatives, having a high electric field around the thin wires but well below the critical values for multiplication in most of the remaining volume, a transition from proportional to streamer regime is also observed at high voltages, but in most cases the streamer propagation stops in the decreasing field well before reaching the cathode. The limited streamer regime has been widely exploited for obtaining conveniently large signals in wire counters [30] and has as sole shortcoming a modest rate capability.

\section{PREVIOUS OBSERVATIONS WITH MICRO-PATTERN DETECTORS}

The appearance of discharges on approaching some critical voltage is a permanent struggle with gas proportional counters, and has been intensively studied for many detector designs and in a wide range of operating conditions aiming at the optimization of performances.

Three major mechanisms leading to the onset of a discharge can be distinguished:

a) Spontaneous breakdown, in absence of radiation, above a critical voltage. This appears to be geometry and position-dependent, particularly in micro-pattern detectors, revealing the essential role of the manufacturing quality and of local defects. An example of distribution of discharge voltage in absence of radiation across a micro-strip chamber is shown in Fig. 1 [31, 32]. For the measurement, realized with a $10 \times 10 \mathrm{~cm}^{2}$ MSGC plate with thin chromium anode and cathode strips at $200 \mu \mathrm{m}$ pitch manufactured on diamond-like coated glass [33], groups of 16 strips were sequentially activated with all others left floating; each entry in the histogram represent the discharge voltage threshold for one group. The range is rather wide, a typical feature of MSGCs, due to manufacturing imperfections and/or presence of unsolicited residues introduced during assembly. The overall performance of the plate is obviously determined by the worst groups, discharging at a gain around $10^{3}$, while most of the remaining area would permit gains almost an order of magnitude higher to be reached. Usually, only a few strips are found to be defective within the group, and can be disconnected from operation; this is often done to improve the general behavior of the detector. It can also be seen that, with a suitable selection of the active area, a wide range of performances can be covered; this clearly demonstrates the advantages and dangers intrinsic in the exposure to radiation of small areas only to characterize a detector.

b) Rate-induced breakdown. Contrary to classic multiwire chambers, where the outcome of a high counting rate is to reduce the gain, due to the field modification induced by accumulating positive ions, in high-rate micro-pattern devices the most important effect of rate is a reduction of the maximum operating voltage due to the onset of discharges. Given the very fast clearing of ions in the small gaps and high fields typical for the devices (few $\mu \mathrm{s}$ ), simple arguments relying on a charge built-up due to the overlap of avalanches do not seem to hold, and the physical reasons for the rate dependence of the discharge point are still subject of speculations. Some authors have suggested the presence of long-lived excited states 
produced in the gas or, according to recent evidence, more likely on the electrode surface [34].

Because of the irreversible damaging effects of discharges on thin anode strips, scanty data exist on the rate dependence of the maximum voltage that can be reached in MSGCs. Recent measurements indicate a decrease of the maximum gain, as compared to the low-rate value, by a factor of around two at $10^{5} \mathrm{~Hz} / \mathrm{mm}^{2}$ [35]. Similar results have been obtained with a micro-gap chamber [22]. Both measurement were realized exposing a small (few $\mathrm{mm}^{2}$ ) area of the detectors to a collimated $6 \mathrm{keV} \mathrm{X-}$ ray beam.

Systematic exposure of individual groups of strips, covering one at a time the whole active area of a MSGC, exhibits also a position-dependent discharge voltage under high-flux continuous irradiation (Fig. 2) [31]. By comparison with Fig. 1, one can see that the average maximum voltage under strong irradiation is decreased by around $50 \mathrm{~V}$, with a corresponding decline of the useful gain. The maximum gain for the full device is of course determined by the worst group; even inhibiting several strips in the particularly bad groups, the average gain before discharge does not exceed a few thousand, quite a marginal value for efficient detection of minimum ionizing particles.

c) Heavily ionizing tracks. Exposure to particles with high ionization power results also in a considerable decrease of the maximum safe operating voltage. Various methods have been used to characterize this process, and will be described in the next section. The plot in Fig. 3 (deduced from unpublished data of the Heidelberg group ${ }^{1}$ ) shows the discharge probability, defined as the fraction of signals with exceedingly large amplitude, as a function of cathode voltage for a MSGC exposed to an internal ${ }^{220} \mathrm{Rn} \quad \alpha$ emitter. The detector was a $10 \times 10 \quad \mathrm{~cm}^{2}$ device manufactured with chromium strips on a diamond-like coated glass with reduced surface resistivity $\left(\sim 10^{14} \Omega\right.$ /square). Fig. 4 shows measurement realized at CERN with the MSGC described in Ref. [36], similar in construction to the previous, and exposed locally to an external ${ }^{241} \mathrm{Am} \alpha$ source. In both measurements, the discharge threshold is met at a gain between 2 and $3.10^{3}$.

In a recent work, the authors of Ref. [32] have compared the performance of a standard MSGC, alone and with the addition of a gas electron multiplier as pre-amplifying stage, under identical conditions of irradiation. The results of the simultaneous exposure to a high $\mathrm{X}$-rays flux $\left(\sim 10^{4} \mathrm{~Hz} \mathrm{~mm}^{-2}\right)$ and to the internal $\alpha$ source are summarized in Fig. 5, providing the distribution of discharging groups as a function of the MSGC cathode voltage, as well as the corresponding gain curves (measured at low rates) without and with a moderate added preamplification (by a factor of 50). Remarkably, the MSGC discharge voltage is almost independent of the GEM gain, resulting in a considerable increase of the safe gain for the cascaded operation. Even including a group seriously damaged during the study (indicated by the arrow), the operation at gains up to ten thousand is still possible. This observation has led to the adoption of the MSGC+GEM technology by the HERA-B experiment [17].

\footnotetext{
${ }^{1}$ Provided by C. Besch, Heidelberg University
} 


\section{EXPERIMENTAL SET-UP AND PROCEDURES}

All measurements reported here on various detectors have been performed as far as possible in identical conditions and, whenever allowed by the size of the detector, exposing to radiation similar active volumes; in all devices, the thickness of the sensitive gap (the conversion and drift volume) was around $3 \mathrm{~mm}$.

After powering the device and a verification of a correct behavior with the help of low intensity sources, the following steps were performed:

a) Absolute gain calibration. Exposing a limited area of the detector to a constant and uniform flux of X-rays from a generator, the anode and cathode currents are recorded in a wide range of operating voltages. In multiple electrode devices, as for example the double GEM detector, the current is measured also on the additional electrodes. For the (grounded) anode, the current $I_{A}$ is derived from the potential drop on a high value load resistor, while for the powered electrodes it is directly provided by a reading on the $\mathrm{HV}$ units $^{2}$. Subsequently connecting the anodes (usually wide groups of strips) to an amplifier and to a low threshold discriminator, the corresponding counting rate $R$ is measured. The absolute anodic gain is then computed as:

$$
M_{A}=\frac{I_{A} w}{R e E_{x}}
$$

where $E_{x} / w$ is the number of ion pairs released in the gas by each X-ray conversion $(\sim 220$ for $6 \mathrm{keV})$, and $e$ the electron charge. The expression provides the effective, or usable gain, contributing to the signal detection; in GEM structures, the real gain can be larger by an amount that depends on the applied fields, but does not exceed about $20 \%$ in normal operating conditions [37, 38].

b) Full volume $X$-ray irradiation. Placed at a convenient distance $(\sim 50 \mathrm{~cm})$ from the $\mathrm{X}$-ray generator, the active area of the detector is irradiated with a roughly uniform radiation flux. A thick brass absorber with an opening adjusted to the detector size $\left(\sim 80 \mathrm{~cm}^{2}\right.$ for the $10 \times 10 \mathrm{~cm}^{2}$ devices) is placed in front of the detectors to prevent possible edge problems. The flux uniformity, measured independently with the help of a small collimator, is better than $10 \%$ over the irradiated area; the value of the flux is deduced from the measured currents and the known absolute gain (see above). For each setting of the X-ray flux, the detectors' operating voltage is increased progressively, recording the anodic current, until instabilities or discharges are encountered.

c) Exposure to heavily ionizing particles. Opening the gas flow to a bypass containing a thorium oxide compound ${ }^{3}$, the mixture is enriched with radon ${ }^{220} \mathrm{Rn}$ whose main decay mode (with $54 \mathrm{sec}$ lifetime) produces $\alpha$ particles of $6.4 \mathrm{MeV}$. Depending on the distance from the generator, the gas flow and the detector geometry, we record between a few and a few tens decays per second and $\mathrm{cm}^{3}$ of active volume. The pulse height spectrum directly recorded at moderate gains on a wide group of anodes, without an

\footnotetext{
${ }^{2}$ We have used for this purpose CAEN N471A high voltage modules with $1 \mathrm{nA}$ sensitivity.

${ }^{3}$ In the form of a mesh used for light enhancements in gas flame lamps (Welsbach mantle).
} 
electronic amplifier to avoid saturation, is shown in Fig. 6; the energy scale has been (approximately) calibrated by comparison with the charge detected on soft X-rays. The spectrum has a peak around $500 \mathrm{keV}$, and an exponentially decreasing tail extending to several $\mathrm{MeV}$.

In all measurements, the most delicate issue is the definition of the discharge limits and rates. As indicated in the previous section, several methods have been used by different authors and a comparison of results is not always straightforward. For the present study, we have adopted the rather extreme position of defining a discharge as an event causing an overload of the current-limited power supplies, set at a threshold of about ten times the average normal current, with the consequent temporary inhibition of the detected normal signals. To avoid damages due to sustained discharges, a dead time of several minutes has been built-in after each discharge before restoration of the voltages. Despite its roughness, this definition of discharge is in fact the closest to represent the practical limits in the use of a detector. To avoid distortions caused by the power supply recovery time, and also possible damages to the more delicate structures, the measurement is suspended when the discharge rate exceeds a few counts per minute. We have verified that this definition gives results virtually coinciding with those obtained with the alternative method of counting pulses exceeding a pre-set large threshold, or precursors, typically an order of magnitude higher than the largest normal signals.

In most detectors, we have observed a rather sharp transition from the condition of no discharge to the onset of instabilities; with reference to Figs. 4, one may define as "discharge limit" the voltage just below the first non-zero count (580 V in the figure), and "maximum gain before discharge" the corresponding value of gain. In most plots, this value is indicated by a dashed vertical line. Note however that, in view of the very low discharge rate often recorded at the lowest voltages, these indicative values depend on the time allowed for each measurement, and a complete discharge probability curve such as those shown is a more realistic representation of the process.

\section{EXPERIMENTAL RESULTS}

Systematic measurements of discharge limits under high-flux X-ray exposure and internal $\alpha$ decays have been performed with a collection of recently developed detectors; Table 1 provides schematics and summary of parameters for the devices tested. Some of the detectors were built at CERN, others were provided by collaborating institutions, as indicated later in the text. Whilst we have attempted to operate all detectors in similar conditions, sometimes this was not possible or desirable; for example, the active volume for some devices (e.g. the micro-dot) was considerably smaller, and the filling gas (a mixture of argon and carbon dioxide for all GEM-based devices) was changed to use mixtures preferred by the developers of the micro-dot, micromegas and the advanced passivation MSGC. In general, a wider investigation on the effect of the gas choice on the performance of the detectors would be desirable; however, 
measurements with GEM in different mixtures (argon with carbon dioxide, argon-DME and neon-DME, see later) yielded essentially the same results. Also, taking into account the previous discussions on the nonuniformity of response, some variability in the results may have been introduced by the uneven quality of the detectors themselves. In comparing the measurements, one should consent to a spread, within the same class of devices, of perhaps a factor of two in the observed values of limiting gain. Nevertheless we consider that the major conclusions of this study are general enough to be only marginally affected by this variability.

\subsection{Micro-strip gas chamber: standard}

Measurements with this device have already been reported, and have not been repeated; Figs. 1 to 4 provide a summary of previous results. One should note that a discharge in the delicate MSGC structures often results in permanent damage, affecting the performances of the detector; this is particularly true if a low melting point metal such as gold is used for the strips. The outcome of the measurements depends, more than with any other, on the previous history of the device.

\subsection{Micro-strip gas chamber: advanced passivation}

A technology for protecting the cathode strips edges with a thin polyimide coating has been recently developed, with the aim of increasing the voltage required for the onset of discharges [20]. We have tested a $10 \times 10 \mathrm{~cm}^{2}$ MSGC plate with the so-called advanced passivation, manufactured with gold strips on a support coated with a thin layer of electron conducting glass ${ }^{4}$. The operating conditions were those recommended by the authors: a high value of drift field $(3.7 \mathrm{kV}$ on 3.3 $\mathrm{mm}$ ) and a gas filling of neon and dimethylether in the approximate proportions 40-60. The detector was irradiated with a collimated high flux of X-rays $\left(\sim 2.10^{4} \mathrm{~Hz} \mathrm{~m} \mathrm{~m}^{-2}\right.$ over $\left.2 \mathrm{~m} \mathrm{~m}^{2}\right)$ measuring the anode current; the results, Fig. 7 , show indeed that rather high gains, close to $10^{4}$, can be reached. When exposed however to the internal $\alpha$ source, the detector suffers from a considerable decrease in the maximum voltage before discharge, as shown in the figure. At a gain of 1700, the nominal value for full efficiency in the CMS detector [39], reached in our test at a cathode voltage of $530 \mathrm{~V}$, the discharge probability is $0.4 \%$, actually larger than the one observed at the same gain in standard MSGCs (Figs. 3 and 4).

Our results contradict the observations of the authors of Ref. [20], who found a much higher limit of alpha-induced discharge in nominally identical conditions. It should be mentioned, however, that the MSGC plate used had some previous history, and about $20 \%$ of the anodes had been disconnected, being shorted or discharging; we intend to repeat the measurements with a virgin plate when available. Nevertheless, the fact that in presence of a low radiation flux the active part of the detector could safely hold more than $620 \mathrm{~V}$ is a clear indication of the fundamental physical nature of the charge-induced discharge process, and of the

\footnotetext{
${ }^{4}$ Produced by Alenia, Italy, and provided by IIHE ULB-VUB Brussels.
} 
ineffectiveness of the advanced passivation in preventing the transition. To confirm our observation, at the end of the exposure the detector was flushed clean and powered again (after floating several damaged strips); at $580 \mathrm{~V}$, no discharge was observed in a 10-hours long run, confirming the role of the ionizing tracks in inducing discharges.

\subsection{Gas electron multiplier: standard}

Many exposures have been carried out with GEM devices, covering a wide range of drift and induction fields; they provided similar results, and only the most significant is reproduced here. All GEM meshes were of the most recently developed high-gain model, produced with a double mask processing and having quasi-cylindrical holes $80 \mu \mathrm{m}$ in diameter at the metal sides [37, 40]. As a filling gas we have used argon- $\mathrm{CO}_{2}$ in the volume percentage 70-30, a convenient mixture for the use in large detectors, even though certainly not the best for obtaining high gains [32]. Fig. 8 shows, as a function of GEM voltage and at increasing rates, the current measured on a group of anode strips (covering $100 \times 5 \mathrm{~mm}^{2}$ ) whilst irradiating the whole active are of the detector with X-rays. The scale on the right provides, for the lowest curve, the corresponding values of effective gain. The gain curves are exponential and parallel within the errors, demonstrating the excellent rate capability of the GEM detector. The last point on each line corresponds to the voltage value reached just before discharge, and the dashed curve represents the boundary for stable operation. The maximum stable gain, around 8000 at low rates, is reduced by a factor of two at a flux of $10^{4} \mathrm{~Hz} \mathrm{~mm}^{-2}$ over the whole active area.

In Fig. 9, one can see the effect of adding the internal $\alpha$ emitter; gain and discharge probability are given as a function of GEM voltage. Realized with a higher field in the induction gap, the gain curve is slightly shifted, compared to the previous, to lower voltages, due to the increased collection efficiency [37]. Stable up to a gain of about 1500, the detector exhibits a low but quickly increasing rate of discharges above that value.

Several attempts have been made to improve the maximum gain with a different choice of the gas mixture, having a priori better quenching properties than $\mathrm{A}-\mathrm{CO}_{2}$. While moderately larger gains could indeed be obtained, for example in DME-rich mixtures, the gain at discharge on exposure to heavily ionizing tracks appears to be almost invariant. As an example, Fig. 10 shows the results obtained with neon-DME, a mixture reported to permit high gains in MSGCs due to a strong suppression of photon emission in the avalanches [41]. As before, a fast increasing $\alpha-$ induced discharge probability at gains above 1500 is observed.

\subsection{Gas electron multiplier: conical}

The characteristics of GEM devices with conical holes, conveniently manufactured with a single-mask processing, have been described before [40]; because of peculiarities in their performances (a more pronounced charging-up, and limitations to the values of external fields) they have not yet been used extensively. 
We have employed for this measurement a gain-limited early prototype, with rather wide hole diameters of 90 and $45 \mu \mathrm{m}$ on the two sides respectively. Fig. 11 summarizes the behavior of a conical GEM with PCB readout, mounted in two configurations: electrons drifting from the narrow to the wide side $(\mathrm{N}->\mathrm{W})$ and the reverse $(\mathrm{W}->\mathrm{N})$. As expected for this design, the maximum gain that can be reached at low irradiation rates is moderate. The internal $\alpha$ emitter reduces this gain further, with a slight preference for the $\mathrm{N}->\mathrm{W}$ configuration (gain 2000 as against 1000 before discharge). Because of the different field configuration and charge sharing in the two configurations, the real gains at discharge (as against the effective ones shown in the figure) are actually rather close.

\subsection{Micro-CAT (or WELL) detector}

Recently introduced, the Micro-CAT [8], micro-groove [9] and WELL [10] detectors are in fact variants of the original CAT device [4], realized with the more sophisticated etching technologies developed for GEM. We have manufactured a WELL device replacing the open holes on the back side of a standard GEM with collecting strips constituting anodes for the multiplying structure. The response of the detector under $\alpha$ irradiation is shown in Fig. 12; the maximum operating voltage before discharge corresponds to a gain of 2000. Note that in this device, and unlike the standard GEM detector, all the avalanche charge is collected, and the effective and real gains coincide; nevertheless, because of the ballistic deficit intrinsic in devices operated in the avalanche mode, the signal detected by a fast amplifier on the anodes is actually smaller.

\subsection{Micromegas}

To realize this measurement we have obtained a commercial device already extensively tested ${ }^{5}$. In order to get consistent results, the filling gas was argon-isobutane in the volume percentages 90-10, as recommended by the providers. Fig. 13 summarizes the results of the exposure to an increasing X-ray flux, and Fig. 14 shows the response to the internal $\alpha$ emitter. All X-ray measurements were realized irradiating most of the active area, with the exception of the highest rate where the exposed area was reduced to $\sim 1 \mathrm{~cm}^{2}$ to elude problems due to the potential drop on protection resistors. In agreement with the original observations [6], very high gains could be achieved, even though a decrease in maximum gain is observed at the highest rates.

Remarkably however, and much as in all previously described devices, introduction of the ${ }^{220} \mathrm{Rn}$ emitter reduced the maximum gain before discharge at a value around 2000. Note that, while the mechanical structure of the detector is impervious to damage due to discharges, this may not be the case of the sensitive electronics used for the readout of signals on the anode strips, particularly in view of the large capacitance of the detector .

\footnotetext{
${ }^{5}$ Manufactured by EURYSIS Mesures, France and provided by ULB Bruxelles.
} 


\subsection{Micro-dot chamber}

The micro-dot tested was a small size standard device developed and provided by the Liverpool group [3, 42]. Consisting of individual anodic dots, $24 \mu \mathrm{m}$ in diameter, centered in a double annular structure (a cathode and an intermediate field ring), the detector was active only over a small area $\left(3.6 \mathrm{~cm}^{2}\right)$ with all anodes interconnected, and was operated, as recommended by the authors, with an argon-dimethylether 70-30 mixture, and at a moderate drift voltage $(1.2 \mathrm{kV}$ over $3 \mathrm{~mm})$. Fig. 15 shows the excellent gain characteristics, as a function of cathode voltage, measured at an X-ray flux around $10^{4} \mathrm{~Hz} / \mathrm{mm}^{2}$. Remarkably, and in opposition to all other devices tested, introduction of the $\alpha$ emitter did not result in any sign of discharge, up to gains of $10^{4}$; at this point, and short of reaching $2.10^{4}$, a fatal breakdown resulted in the destruction of the detector ${ }^{6}$. Observation of the output signal at the highest gain revealed no sign of abnormally large pulses due to streamer transitions or discharge precursors; the energy spectra for the Rn source shown in Figs. 5 were actually obtained by direct connection of the micro-dot grouped output to a pulse height analyzer. This suggests that the low field at the cathode surface and the intermediate field ring act as an effective stop against the formation and propagation of streamers; a better understanding of this feature is certainly very interesting also to try and improve other devices.

\subsection{Cascaded detectors: MSGC+GEM}

Operating characteristics of the detector obtained adding a GEM preamplification element to a micro-strip structure have been already discussed in section 3; Fig. 5 provided an example of measurements made in previous studies [32]. The data show that the insertion of a moderate pre-amplification factor ( $\sim 50$ for the quoted measurement) does not significantly change the operational limits of the higher gain device (the MSGC); on exposure to high rates or heavily ionizing tracks, the discharge is observed at around the same voltage for the MSGC, providing therefore a comfortably higher safety margin. Several groups have confirmed these findings [22, 43-45], and the MSGC+GEM device has been adopted for the HERA-B inner tracker after an extensive campaign of experimental exposures to different beam conditions [17, 46].

\subsection{Cascaded detectors: double GEM+PCB}

The combination of two gas electron multipliers in cascade permits very large stable gains to be achieved; in good approximation, and taking into account the (small) transfer losses between the two elements, the overall gain is the product of the two [47, 48]. With a double-GEM device having the geometry described in Table 1, we have measured at increasing $X$-ray fluxes the current on a group of anodes as a function of the voltage difference applied to the two GEMs. Due to the high values of gain and flux, and to limit the voltage drop on the protection resistors, for the

\footnotetext{
${ }^{6}$ Post-mortem optical inspection revealed marks of discharge damage at known weak edge points.
} 
higher fluxes the irradiated area had to be reduced to a few $\mathrm{mm}^{2}$, and the data correspondingly normalized. As shown in Fig. 16, the current measured on the charge-collecting anodes increases exponentially with the voltage applied to the second GEM, for fixed value of the first, and curves at increasing rates are parallel within the measurement errors, demonstrating the absence of surface or space charge distortions. Fig. 17 provides, for the highest irradiation rate $\left(510^{5} \mathrm{~Hz} \mathrm{~mm}^{-2}\right)$ the effective gain as a function of voltage applied to the multipliers; a gain well above $10^{4}$ can be reached in a wide range of potentials, with a maximum up to $10^{5}$. The dashed curve in the figure represents the discharge limit; considering the first GEM to act only as a variable charge injector, the trend of withstanding larger gains (larger amount of charge) when the second multiplier operates at lower potentials supports our presumption of a voltage dependence of the discharge limit. We have repeated the gain measurement with exposure of the detector to the internal $\alpha$ source; in this case, only the upper boundary of stability before discharge has been recorded (Fig. 18). The maximum gain is again reduced by an amount that depends on the gain sharing between the two multipliers; the optimum is an operation with close values of the voltage difference. For large offsets between the two, the characteristic limitations of a single GEM are found.

\section{CONCLUSIONS AND SUMMARY}

This work describes the results of systematic measurements obtained with an exhaustive list of recently developed fast gaseous detectors, in order to assess their performances and reliability in the harsh operating conditions that will prevail in experimental set-ups at high luminosity colliders, namely the simultaneous presence of a high flux of relativistic charged particles and of rarer but heavily ionizing tracks. The general trend emerging from the study of single-stage devices is that, although they differ in the maximum gain reached in a low radiation environment, the difference tends to vanish with an exposure to a high flux of X-rays and to heavily ionizing tracks, emulated in this study by an internal alpha emitter added to the gas flow. In these conditions, a nonnegligible probability of a transition from proportional avalanche to streamer followed by a discharge is observed at proportional gains between one and two thousand. A remarkable exception in this class of devices, the micro-dot detector seems to withstand the highest gains in such conditions.

Taking into account the measured ionization loss distribution (Fig. 6), it can be inferred that the transition begins to occur when the average avalanche size exceeds a $2-3.10^{7}$ electrons, close to the so-called Raether's limit. The low discharge probability at the onset of the process, below one percent of the recorded $\alpha$ particles counting rate, suggests that the transition is an outcome of statistical fluctuations on the avalanche formation, leading from time to time to what the authors of Ref. [14] have appropriately named an "explosive" process. Additional experimental evidence of this type of process is discussed in Ref. [49]. In view however of the presence, in most of the described detectors, of high field regions 
around the edges of the cathode structures, imparting additional gain to electrons released there (albeit with small probability), it is not excluded that the transitions can be actually generated by tracks crossing these regions, a possibility discussed in Ref. [16]. The discharge probability increases exponentially towards higher gains, making the detector unusable and subject to irreversible damages. Attempts to use more quenched gas mixtures did not result in noticeable improvements; this is an indication of the fundamental, charge-density dependent nature of the discharge mechanism, as observed long ago in avalanche counters .

Indications of a dependence of the discharge limit on position and geometry suggest that a more thorough investigation on each device may lead to small, and hopefully cumulative, improvements. The effect of the gas composition should also be examined, although in many cases mixtures considered optimal by the authors have been used. The robustness of some detectors, together with effective protection schemes to limit the damages induced by discharges, permit an albeit marginal operation in benign environments.

Sharing the amplification process between two cascaded devices, a gas electron multiplier followed by a MSGC or by another GEM, results in a shift upwards by at least an order of magnitude of the maximum gain sustainable, both at high rates and under exposure to heavily ionizing tracks. This is an intuitive consequence of the fact that, given the overall gain, each device is operated at a considerably lower voltage; a possible explanation of the observation is in a voltage dependence of the Raether limit, higher for lower operating voltages. This conjecture is supported by measurements with the double GEM detector, in which the first multiplier is used as an injector of variable amounts of charge into the second.

An alternative explanation for the increased limit of divergence has been suggested by some authors ([22] and references therein) as being a consequence of the reduction of charge density in the avalanche induced by the additional spread due to diffusion in the double devices. Recent measurements of cluster size in single and double GEM detectors however do not seem to support this theory, the average avalanche size increasing only by a small amount $(20 \%)$ by the additional multiplication [48]. More work on the subject is certainly required.

The comfortable margin between the gain needed for full efficiency detection of fast particles $(\sim 2000)$ and the maximum gain before discharge in the double devices $\left(>10^{4}\right)$ suggest that this recently introduced family of detector is the most suited for reliable use in high rate experimental setups, with simultaneous presence of a high flux of relativistic charged particles to be detected, and of a considerable background of unwanted, highly ionizing events, as for example proton knock-off by neutrons conversions and production of nuclear fragments.

It should be mentioned that simulation studies show that alpha particles, as used in this work, give an optimistic view of the conditions to be met at LHC [50]. Under these circumstances, and pending a realistic test of the devices at the new collider, it is suggested that more experimental work and a careful analysis to the operational safety margins should be made before the adoption of a detector geometry for the experiments. 


\section{ACKNOWLEDGMENTS}

Two of the detectors used in this work, micromegas and the advanced passivation MSGC, have been provided by W. Van Doninck and S. Tavernier (IIHE ULB-VUB Bruxelles); their contribution is here acknowledged. The technology for manufacturing the GEM electrodes has been developed by A. Gandi and R. De Oliveira (CERN-EST-MT) . The detectors were assembled by M. Delattre and M. Van Stenis in the EP-TA1 group led by A. Placci, whose continuing support for our work is here acknowledged. The wire bonding for the connection of the electrodes to the readout boards have been made by $\mathrm{O}$. Runolffson and K. Muhlemann (CERN-EP-OPAL).

\section{REFERENCES}

[1] A. Oed, Nucl. Instrum. Methods A263 (1988) 351.

[2] F. Angelini, R. Bellazzini, A. Brez, M.M. Massai, R. Raffo, G. Spandre, M.A. Spezziga, Nucl. Instrum. Methods A335 (1993) 69.

[3] S.F. Biagi and T.J. Jones, Nucl. Instrum. Methods A361 (1995) 72.

[4] F. Bartol, M. Bordessoule, G. Chaplier, M. Lemonnier, S. Megtert, J. Phys. III France 6 (1996) 337.

[5] V. Chorowicz, J.-F. Clergeau, D. Contardo, R. Haroutunian, L. Mirabito, S. Muanza, G. Smadja, Nucl. Instrum. Methods A401 (1997) 238.

[6] I. Giomataris, P. Rebourgeaud, J.P. Robert, G. Charpak, Nucl. Instrum. Methods A376 (1996) 29.

[7] F. Sauli, Nucl. Instrum. Methods A386 (1997) 531.

[8] A. Savestrani, H.J. Besch, M. Junk, W. Meissner, N. Sauer, R. Stiehler, A.H. Walenta, R.H. Menk, Study and applications of hole structures as gas gain devices for two-dimensional high rate $\mathrm{x}$-ray detectors, Subm. Nucl. Instrum. Methods (1998).

[9] R. Bellazzini, M. Bozzo, A. Brez, G. Gariano, L. Lantronico, N. Lumb, A. Papanestis, G. Spandre, M.M. Massai, R. Raffo, M.A. Spezziga, The micro-groove detector, INFN PI/AE 98/02. Subm. Nucl. Instr. Methods (1998).

[10] R. Bellazzini, M. Bozzo, A. Brez, G. Gariano, L. Lantronico, N. Lumb, A. Papanestis, G. Spandre, M.M. Massai, R. Raffo, M.A. Spezziga, The WELL detector, INFN PI/AE 98/03. Subm. Nucl. Instr. Methods (1998).

[11] F. Sauli, Nucl. Phys. B 61B (1998) 236-243.

[12] F. Sauli, Gas detectors: recent developments and future perspectives, Wire Chamber Conference Vienna, February 22-26, 1998. CERN-EP/98-51. Subm. Nucl. Instrum. Methods (1998).

[13] T. Beckers, R. Bouclier, C. Garabatos, G. Million, F. Sauli, L. Shekhtman, Nucl. Instrum. Methods A346 (1994) 95.

[14] V. Peskov, B.D. Ramsey, P. Fonte, Nucl. Instrum. Methods A392 (1997) 89.

[15] V. Peskov, B.D. Ramsey, J.J. Kolodziejczak, P. Fonte, Nucl. Instrum. Methods A397 (1997) 243.

[16] R. Bouclier, M. Capeáns, C. Garabatos, G. Manzin, G. Million, L. Ropelewski, F. Sauli, T. Temmel, L. Shekhtman, V. Nagaslaev, Y. Pestov, 
A. Kuleshov, Nucl. Instrum. Methods A365 (1995) 65.

[17] B. Schmidt, Microstrip Gas Chambers: recent developments, radiation damage and long term behaviour, Vienna Wire Chamber Conference WCC98 Feb. 23-27, 1998. Subm. Nucl. Instrum. Methods (1998).

[18] A. Barr, et al., Nucl. Instrum. Methods A403 (1998) 31-56.

[19] B. Boimska, R. Bouclier, M. Capeáns, S. Claes, W. Dominik, M. Hoch, G. Million, L. Ropelewski, F. Sauli, A. Sharma, L. Shekhtman, W.V. Doninck, L.V. Lancker, Nucl. Phys. B 61B (1998) 498-503.

[20] R. Bellazzini, M. Bozzo, A. Brez, A. Cattai, L. Lantronico, N. Lumb, G. Spandre, A. Tsirou, Nucl. Instrum. Methods A398 (1998) 426.

[21] V. Peskov, B.D. Ramsey, P. Fonte, IEEE Trans. Nucl. Sci. NS-45 (1998) 244.

[22] P. Fonte, V. Peskov, B.D. Ramsey, Rate and gain limitations of MSGCs and MGCs combined with GEM and other preamplification structures, Wire Chamber Conference Vienna, February 23-27. Subm. Nucl. Instrum. Methods (1998).

[23] H.S. Cho, W.S. Hong, N. Palaio, J. Kadyk, K.B. Luk, V. PerezMendez, K.S. Joo, J. Vujic, Nucl. Physics B 61B (1998) 258.

[24] L.B. Loeb, Basic processes in gaseous electronics. . Univ. of California Press, Berkeley (1961).

[25] H. Raether, Electron Avalanches and Breakdown in Gases. . Butterworth, London (1964).

[26] J.A. Rees, Electrical Breakdown in Gases. . MacMillan Press, London (1973).

[27] J.M. Meek, Phys. Rev. 57 (1940) 722.

[28] P. Fonte, V. Peskov, F. Sauli, Nucl. Instrum. Methods A305 (1991)

91.

[29] P. Fonte, IEEE Trans. Nucl. Sci. NS-43 (1996) 2135.

[30] E. Iarocci, Nucl. Instrum. Methods 217 (1983) .

[31] B. Boimska, R. Bouclier, M. Capeans, W. Dominik, M. Hoch, G. Million, L. Ropelewski, F. Sauli, A. Sharma, CMS Tracking Note TN/97020 (1997).

[32] R. Bouclier, W. Dominik, M. Hoch, J.C. Labbé, G. Million, L. Ropelewski, F. Sauli, A. Sharma, G. Manzin, Nucl. Instrum. Methods A396 (1997) 50-66.

[33] R. Bouclier, M. Capeáns, G. Million, L. Ropelewski, F. Sauli, T. Temmel, R.A. Cooke, S. Donnel, S.A. Sastri, N. Sonderer, Nucl. Instrum. Methods A 369 (1996) 328-331.

[34] P. Fonte, V. Peskov, B.D. Ramsey, Which gaseous detector is the best at high rates?, Int. Rep. (1998).

[35] Y. Ivanouchenkov, P. Fonte, V. Peskov, B.D. Ramsey, Breakdown limit studies in high rate gaseous detectors, Symposium on Radiation Measurements and Applications Ann Arbor, Michigan, May 12-14. Subm. Nucl. Instrum. Methods (1998).

[36] R. Bouclier, M. Capeáns, A. Di Mauro, M. Hoch, L. Ropelewski, F. Sauli, A. Sharma, L. Shekhtman, CERN CMS-TN/96-018 (1996).

[37] J. Benlloch, A. Bressan, M. Capeáns, M. Gruwé, M. Hoch, J.C. Labbé, A. Placci, L. Ropelewski, F. Sauli, Further developments of the Gas Electron Multiplier (GEM), Wire Chamber Conference Vienna, Feb. 22-26, 
1998. CERN-EP/98-50. Subm. Nucl. Instrum. Methods (1998).

[38] R. Bellazzini, A. Brez, G. Gariano, L. Latronico, N. Lumb, G. Spandre, M.M. Massai, R. Raffo, M.A. Spezziga, Subm. Nucl. Instrum. Methods (1998) .

[39] D. Abbaneo, et al., Nucl. Instrum. Methods A409 (1998) 37.

[40] J. Benlloch, A. Bressan, C. Büttner, M. Capeáns, M. Gruwé, M. Hoch, J.C. Labbé, A. Placci, L. Ropelewski, F. Sauli, A. Sharma, R. Veenhof, IEEE Trans. Nucl. Sci. NS-45 (1998) 234.

[41] F. Angelini, R. Bellazzini, A. Brez, M.M. Massai, R. Raffo, G. Spandre, M.A. Spezziga, Nucl. Instrum. Methods A382 (1996) 461.

[42] S. Biagi, T.J. Bowcock, D. Duxbury, E. Gabathuler, Nucl. Physics B 61B (1998) .

[43] Y. Benhammou, J.M. Brom, J.C. Fontaine, D. Huss, F. Jeanneau, A. Lounis, I. Ripp-Baudot, A. Zghiche, IReS 98-13 (1998).

[44] W. Van Doninck, C. Vandervelde, F. Udo, O. Bouhali, P. Vanlaer, V. Zhukov, GEM and MSGC: Results obtained in the cosmic hodoscope, Wire Chamber Conference Vienna, February 22-26, 1998. Subm. Nucl. Instrum. Methods (1998).

[45] W. Beaumont, T. Beckers, J. DeTroy, V. Van Dyck, O. Bouhali, F. Udo, C. VanderVelde, W. Van Doninck, P. Vanlaer, V. Zhukov, Studies of an MSGC equipped with a GEM grid as a tracking device, Wire Chamber Conference Feb. 23-27, 1998. Subm. Nucl. Instrum. Methods (1998).

[46] M.G. Ziegler, Untersuchungen von Detektorprototypen für das innere Spurkammersystem des HERA-B Experimentes. Thesis at Univ. Heidelberg Univ. Heidelberg (1998).

[47] C. Büttner, M. Capeáns, W. Dominik, M. Hoch, J.C. Labbé, G. Manzin, G. Million, L. Ropelewski, F. Sauli, A. Sharma, Nucl. Instr. Methods A409 (1998) 79.

[48] A. Bressan, J.C. Labbé, P. Pagano, L. Ropelewski, F. Sauli, Beam tests of the gas electron multiplier, Subm. Nucl. Instrum. Methods (1998).

[49] I. Ivaniouchenkov, P. Fonte, V. Peskov, R. Ferreira-Marques, A. Policarpo, IEEE Trans. Nucl. Sci. NS-45 (1998) 258.

[50] M. Huhtinen, Factors to scale highly ionizing particle rates in MSGC irradiation tests to the LHC radiation environment, CMS Note 1997/073 (1997). 


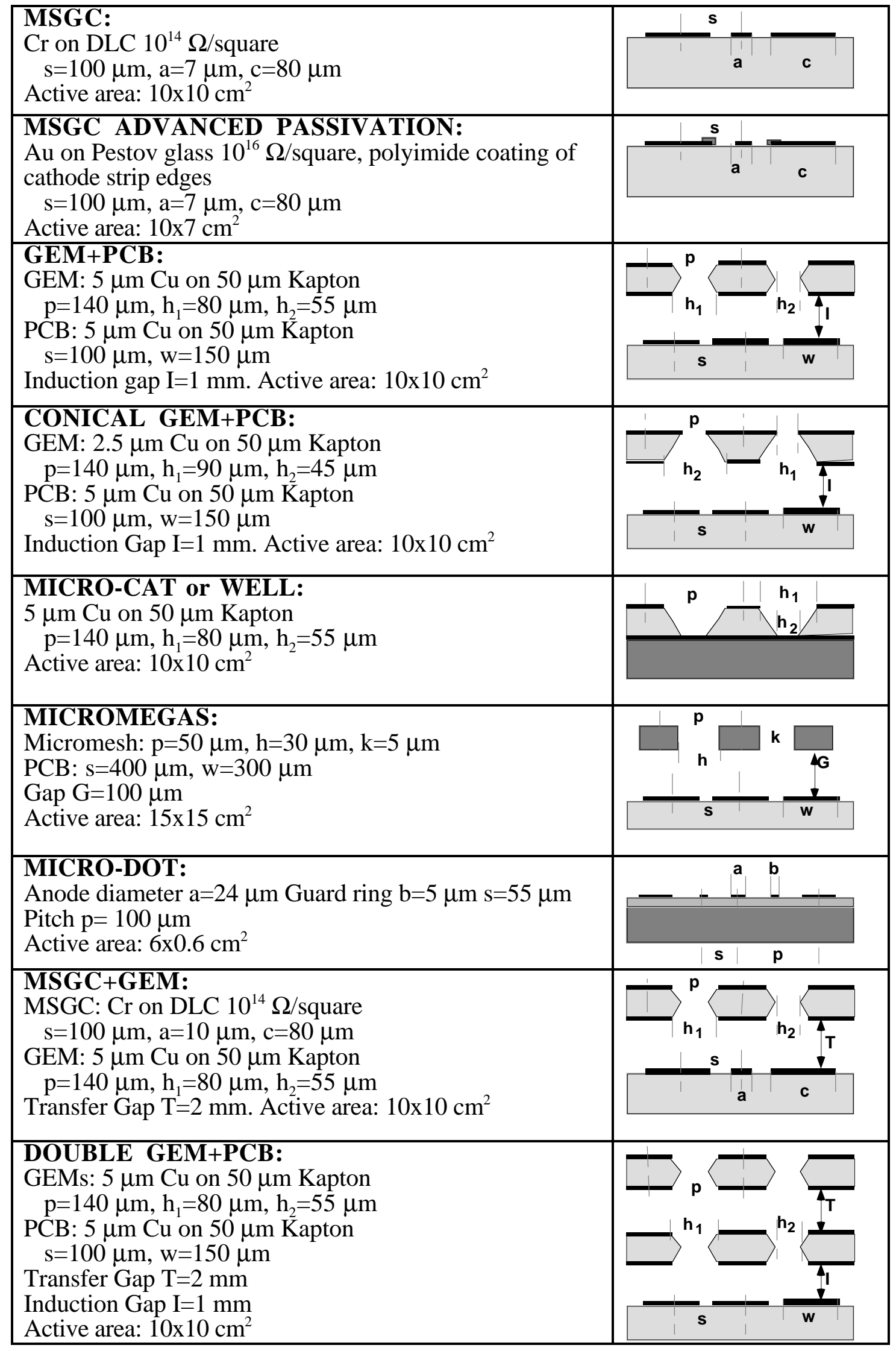

Table 1 


\section{FIGURE CAPTIONS}

Fig. 1: Gain as a function of cathode voltage in a standard MSGC, measured locally and at low rates. The histogram represents the distribution of discharge voltage without radiation for groups of 32 anodes powered in succession.

Fig. 2: Distribution of discharge voltage for the anode groups, under irradiation with soft X-rays at a flux of $10^{4} \mathrm{~Hz} / \mathrm{mm}^{2}$.

Fig. 3: Discharge probability observed in a MSGC exposed to an internal $\alpha$ emitter $\left({ }^{220} \mathrm{Rn}\right)$ by the Heidelberg group.

Fig. 4: Large signals (precursors) probability observed in a MSGC exposed to an external $\alpha$ source by the CERN group.

Fig. 5: Gain (measured at low rates) as a function of cathode voltage of a MSGC without and with the added preamplification of a gas electron multiplier. The histogram represents the distribution of discharge voltage for groups of anodes observed under simultaneous exposure to $\mathrm{X}$-rays $\left(\sim 10^{4} \mathrm{~Hz} \mathrm{~mm}^{-2}\right)$ and to the internal $\alpha$ source.

Fig. 6: Energy loss spectrum of an internal ${ }^{220} \mathrm{Rn} \alpha$ particles source, recorded with the micro-dot chamber.

Fig. 7: Gain as a function of cathode voltage, measured at low rates in a MSGC with advanced passivation, and discharge probability under exposure to the internal $\alpha$ source (gas filling: neon-DME 40-60).

Fig. 8: Current as a function of voltage in the GEM detector with printed circuit readout, at increasing X-ray fluxes. For the lowest curve, the value of gain is given on the right scale. The dashed curve represents the maximum sustainable voltage before discharge.

Fig. 9: Effective gain and discharge probability on the internal $\alpha$ source obtained with the GEM detector with an argon- $\mathrm{CO}_{2}$ gas filling.

Fig. 10: Effective gain and discharge probability with the internal $\alpha$ source observed with the GEM detector in neon-DME.

Fig. 11: Effective gain and discharge probability with the internal $\alpha$ source for two configurations of the conical GEM detector.

Fig. 12: Effective gain and discharge probability with the internal $\alpha$ source for the micro-CAT or WELL detector.

Fig. 13: Anode current as a function of operating voltage measured at increasing $X$-ray rates with the micromegas detector. The right scale gives, for the lowest curve, the corresponding values of gain.

Fig. 14: Gain and discharge probability with the internal $\alpha$ source in the micromegas detector.

Fig. 15: Gain as a function of voltage in the micro-dot detector. No effect of the internal $\alpha$ source has been observed up to a gain of $10^{4}$.

Fig. 16: High rate behavior of the double GEM detector, irradiated over $\sim 3$ $\mathrm{mm}^{2}$ with an increasing X-ray flux. The anodic current is given as a function of voltage in the second GEM, with the first kept constant.

Fig. 17: Effective gain of the double GEM detector at high X-ray flux (5 $10^{5}$ $\mathrm{Hz} \mathrm{mm} \mathrm{m}^{-2}$ ) as a function of voltage on the second GEM, for several values of the first. The dashed curve shows the maximum gain envelope.

Fig. 17: Effective gain as a function of voltages in the double GEM detector exposed to the internal $\alpha$ source. The dashed line is the discharge limit. 


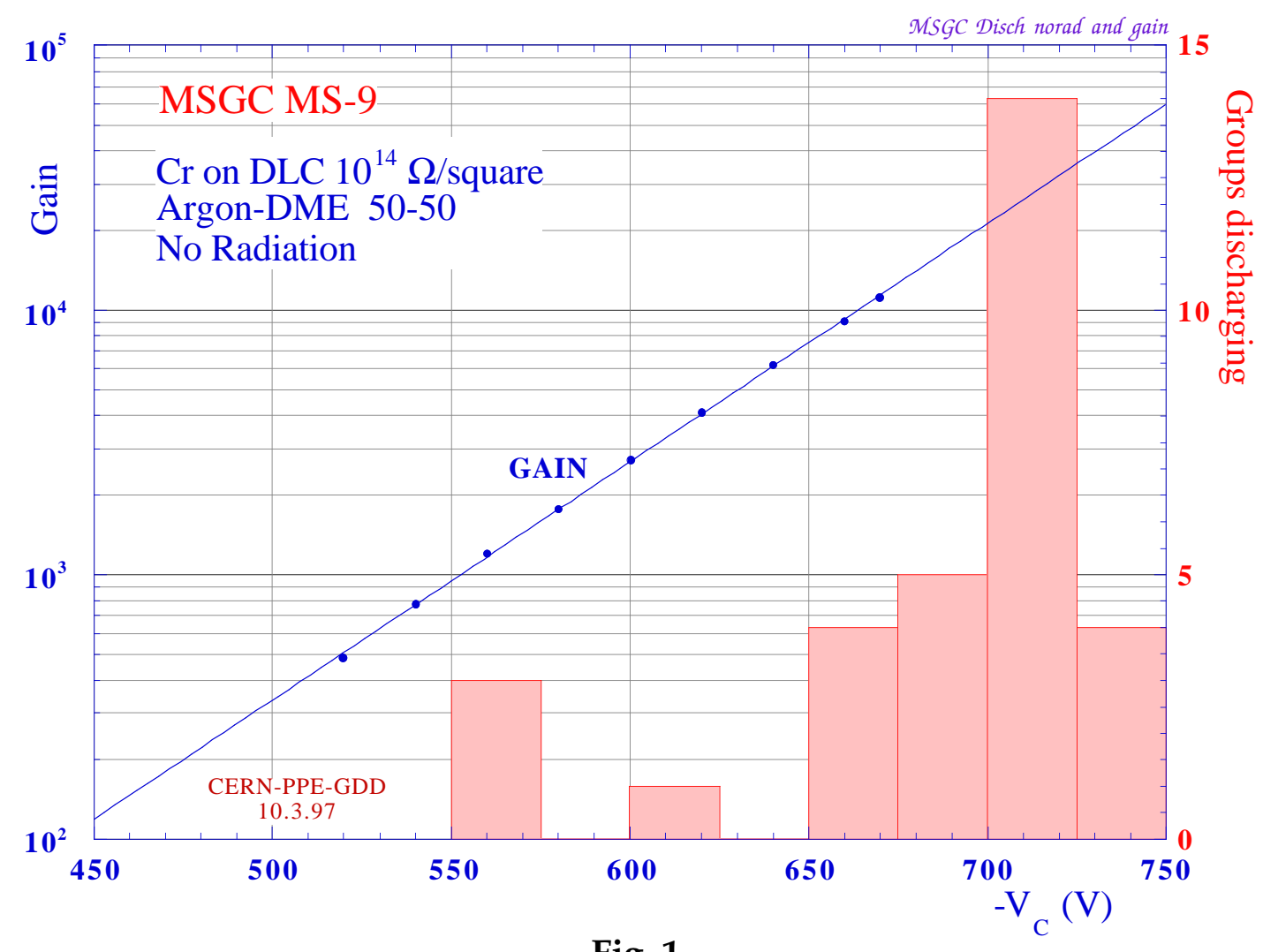

Fig. 1

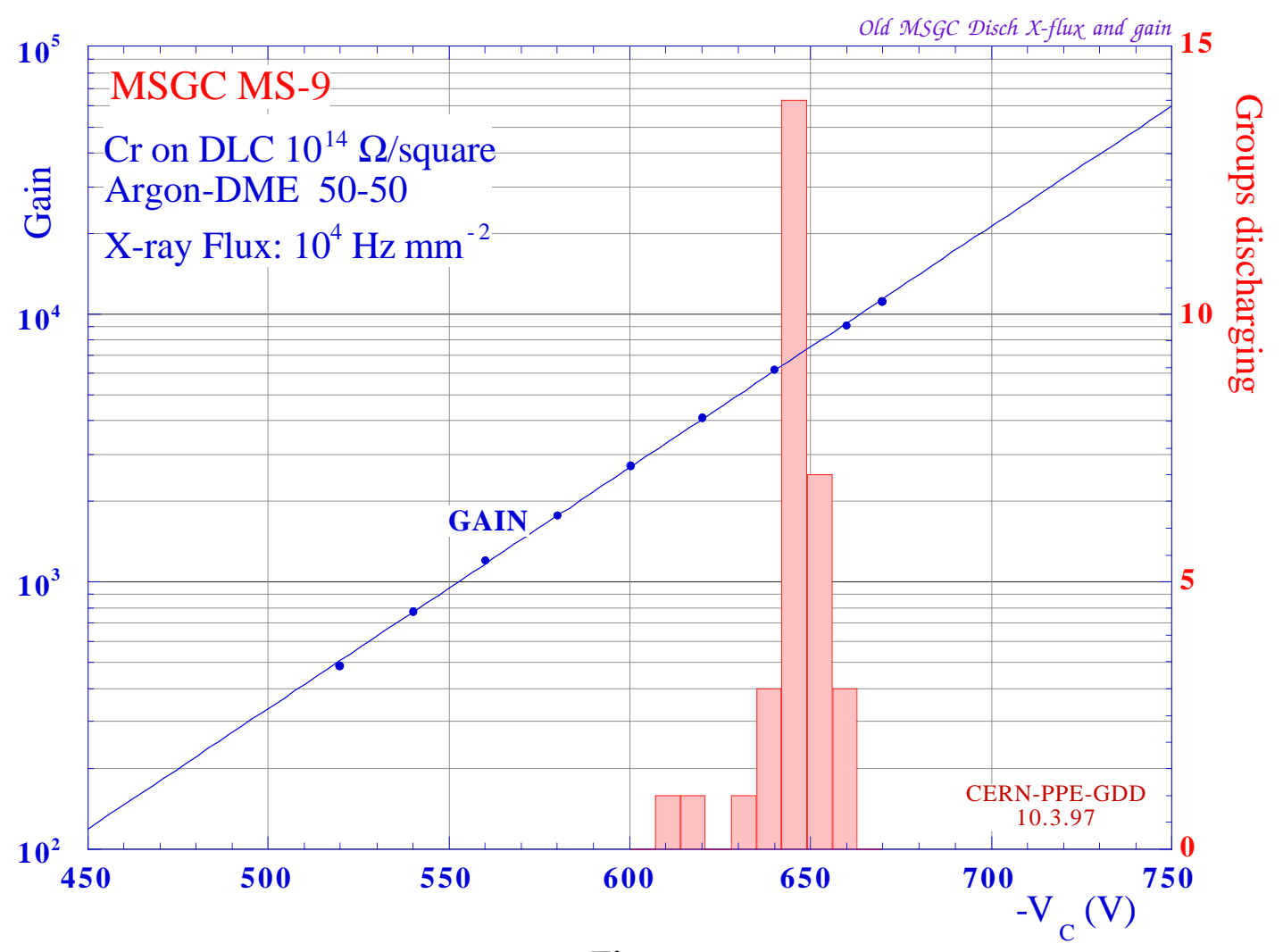

Fig. 2 


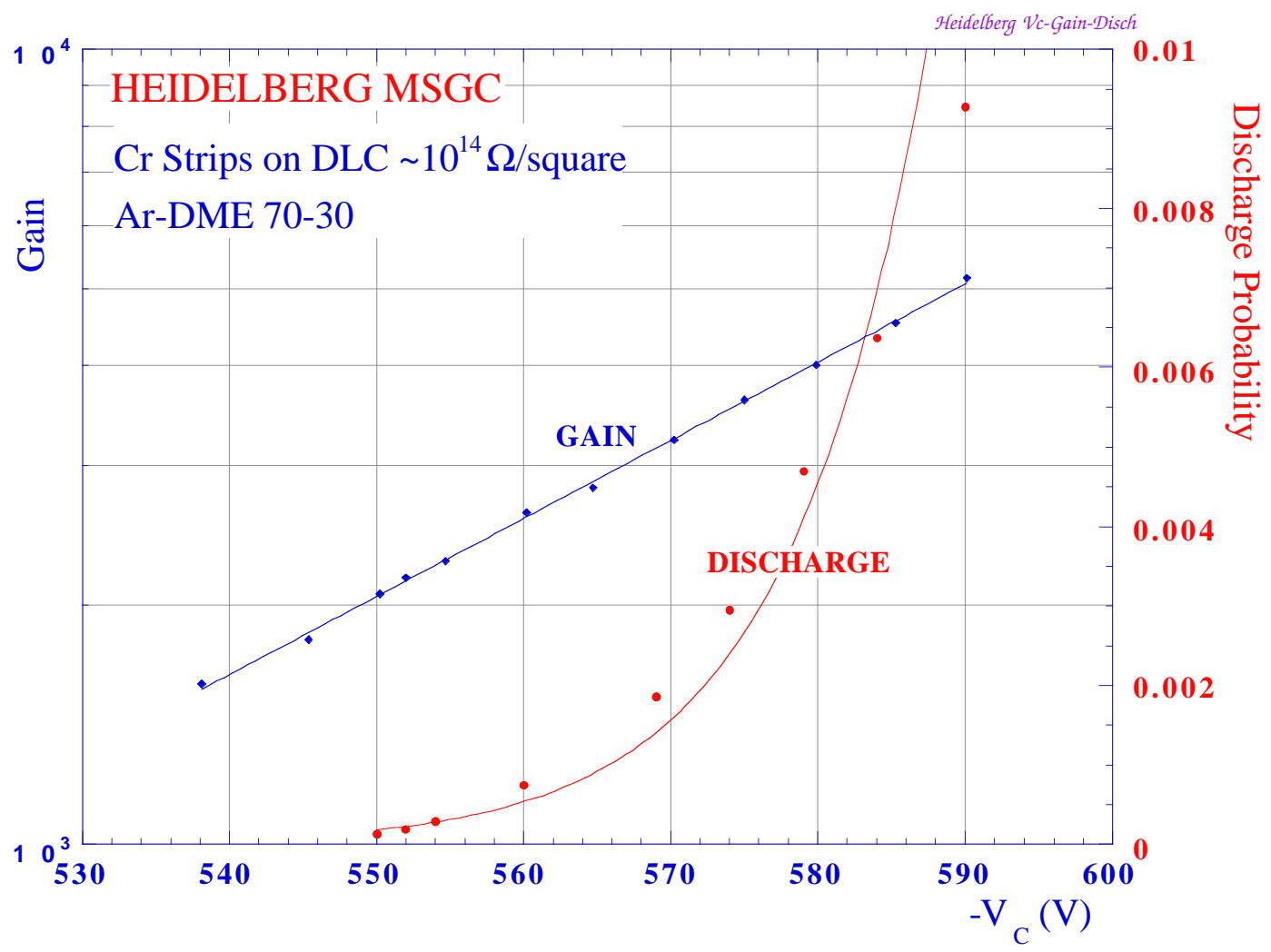

Fig. 3

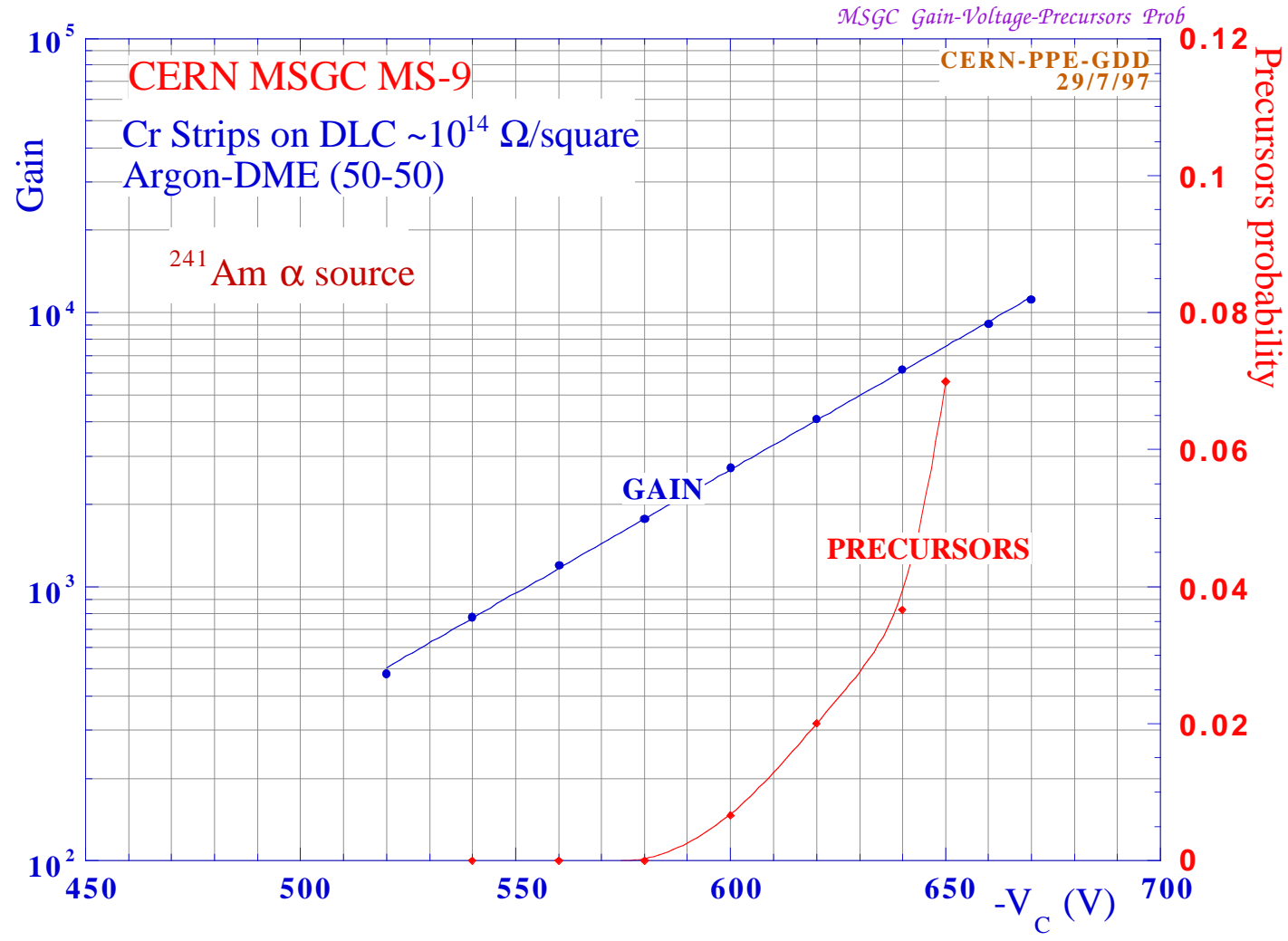

Fig. 4 


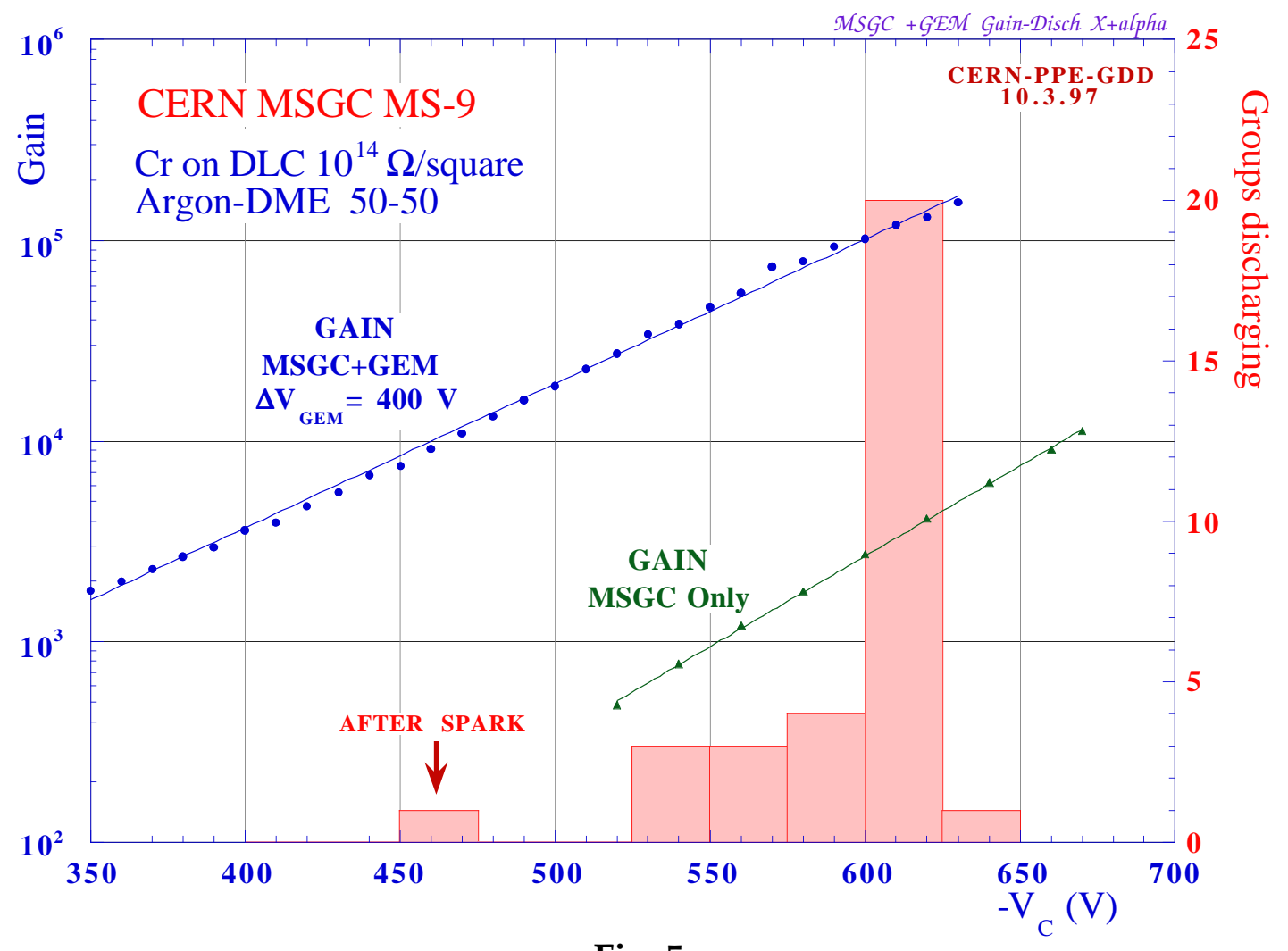

Fig. 5

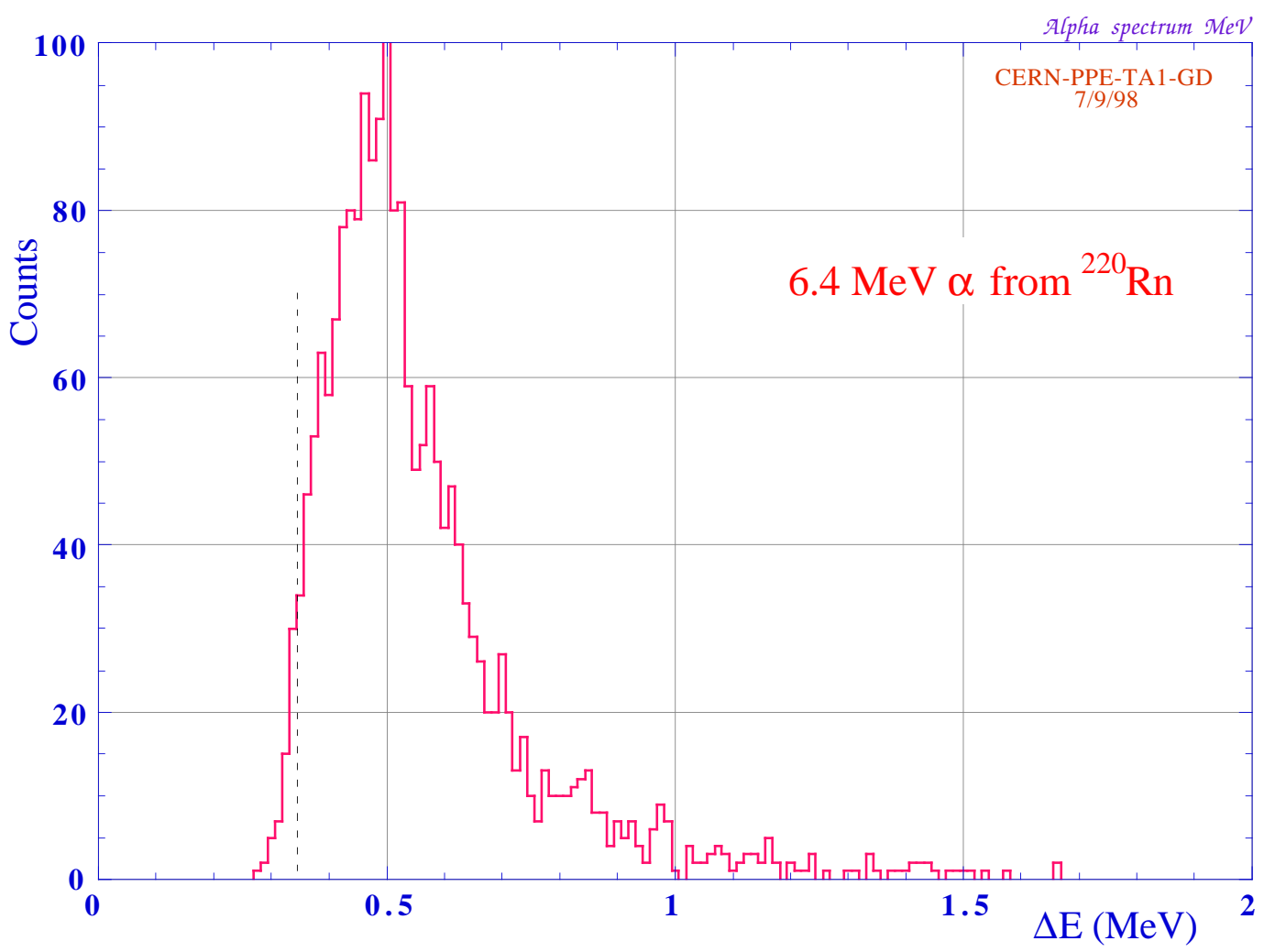

Fig. 6 


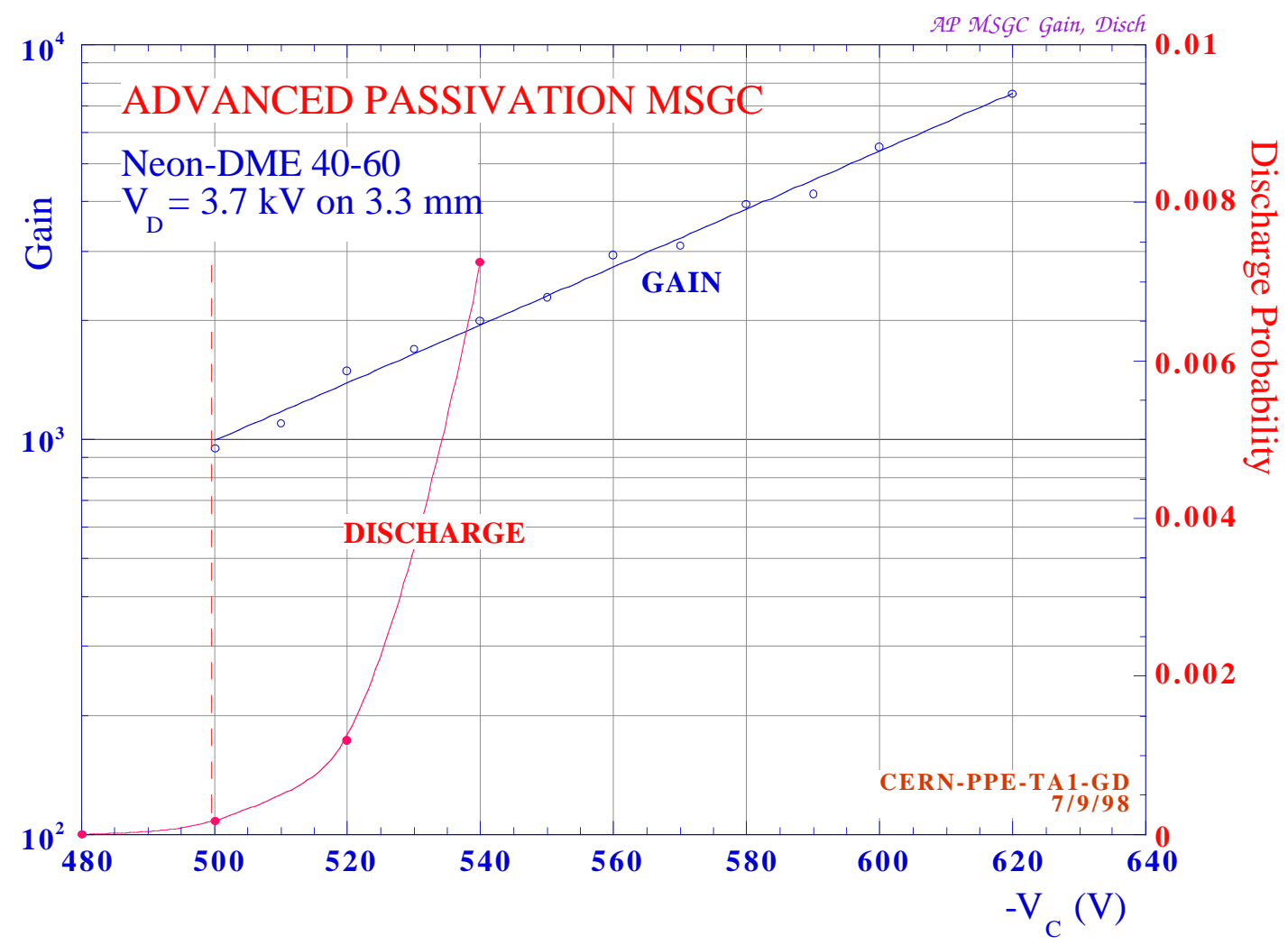

Fig. 7

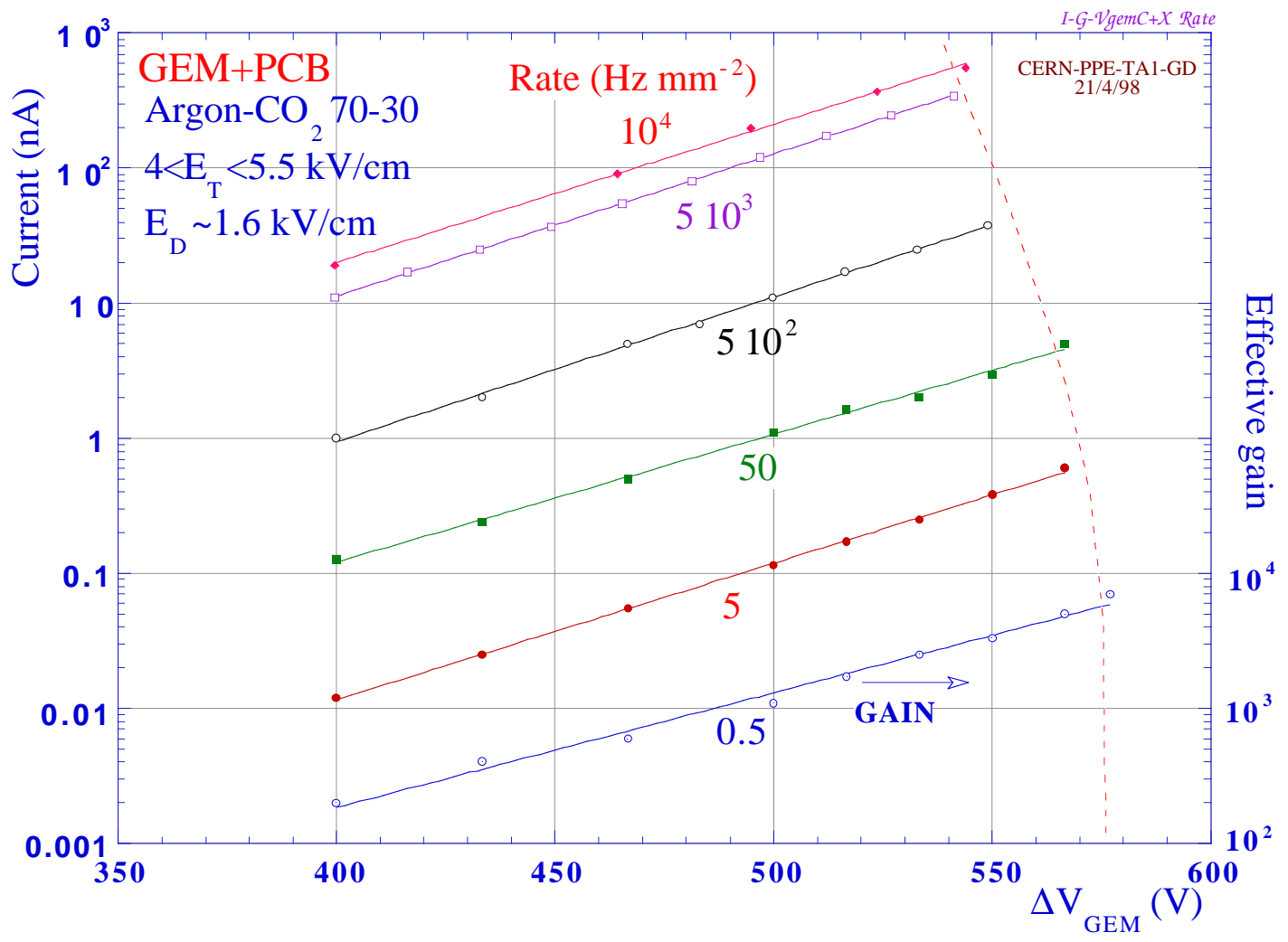

Fig. 8 


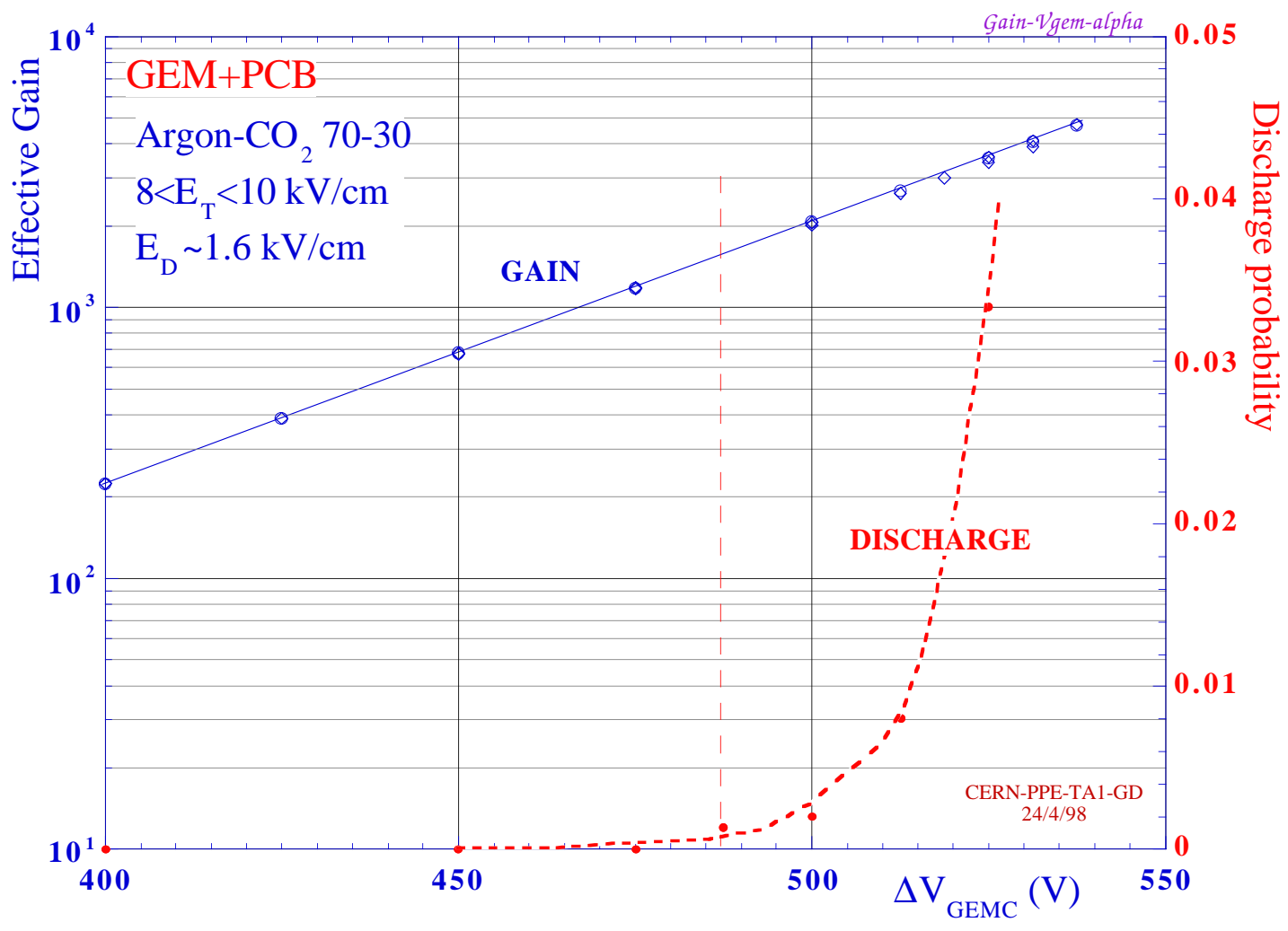

Fig. 9

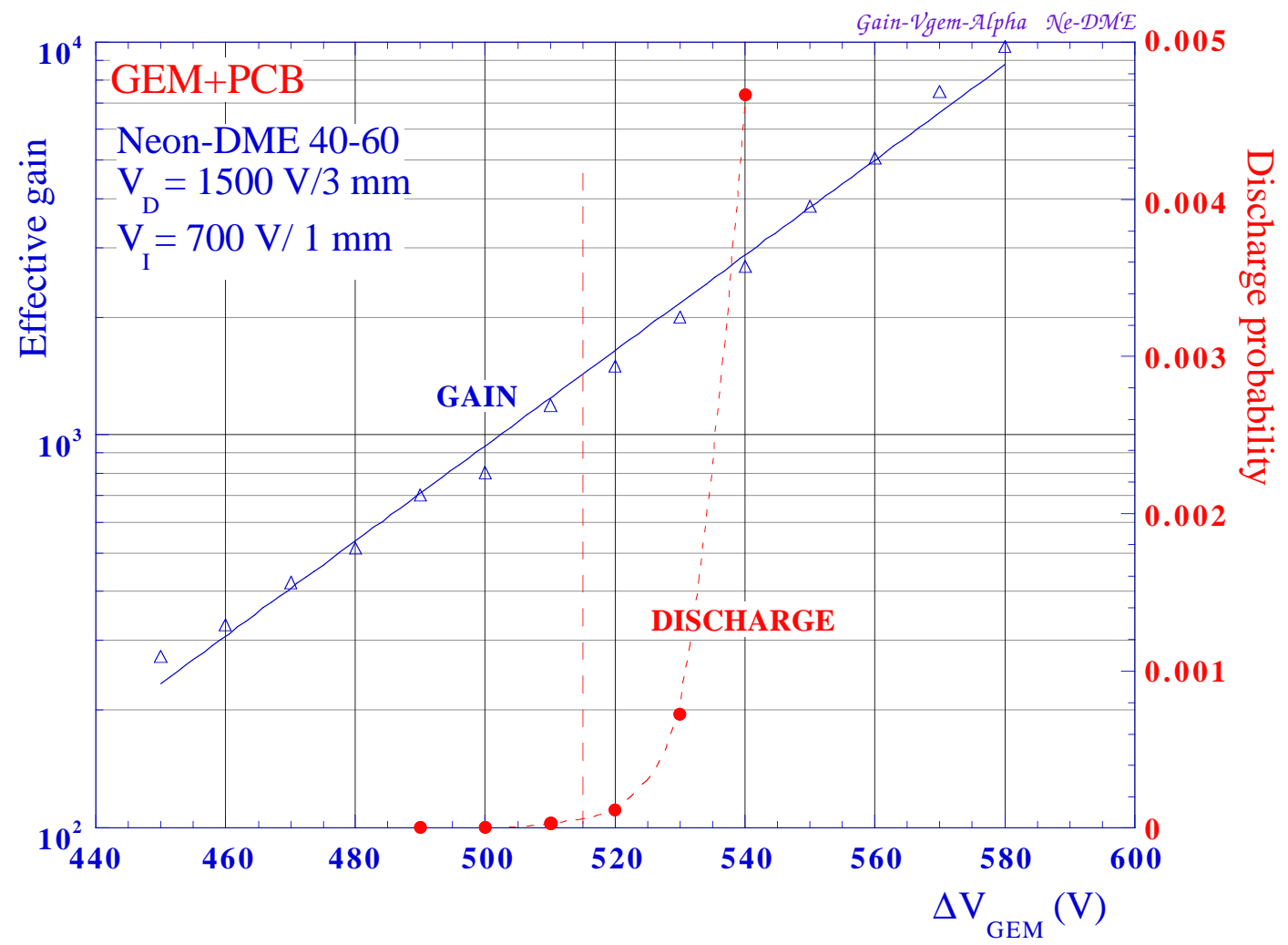

Fig. 10 


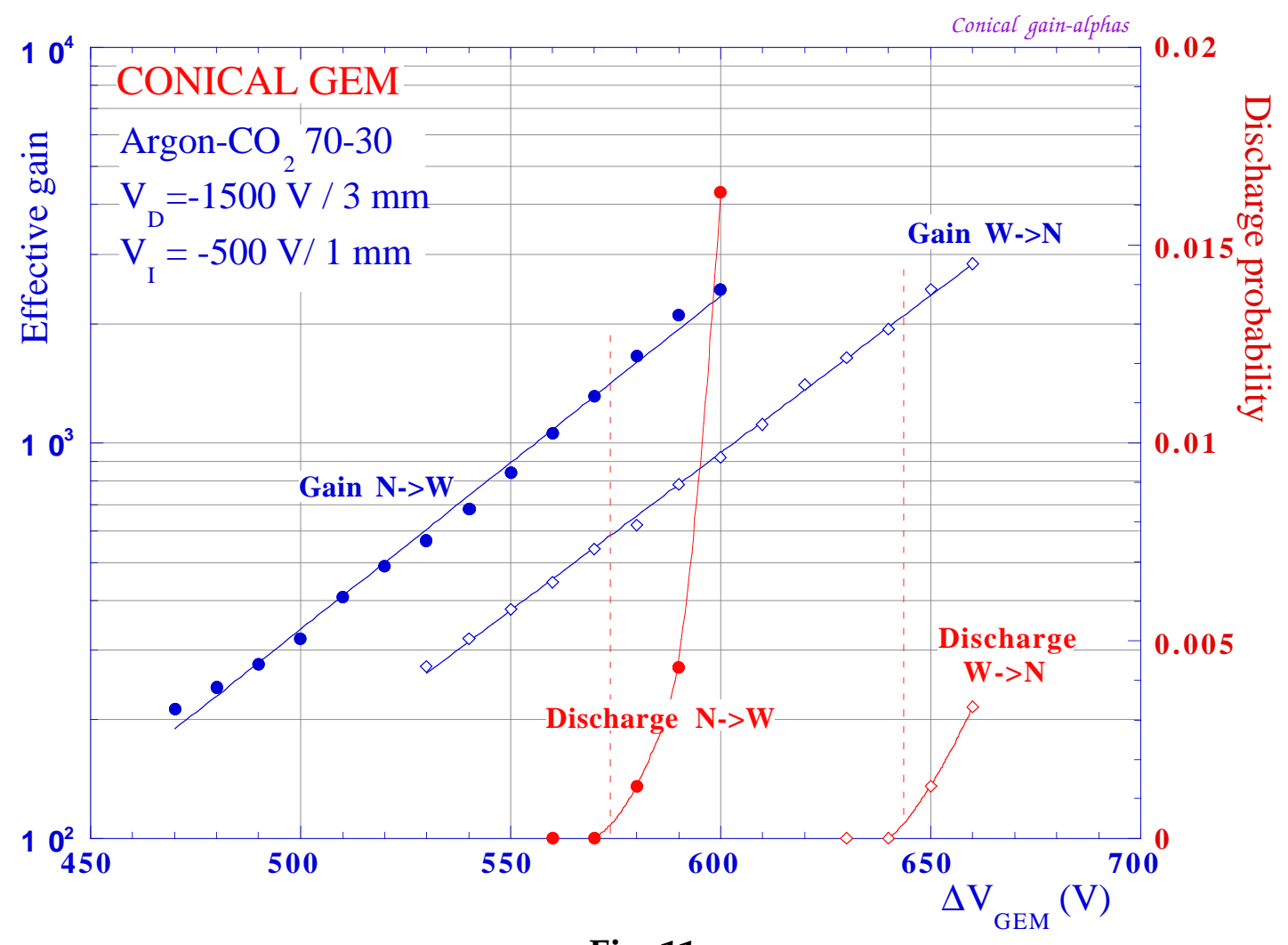

Fig. 11

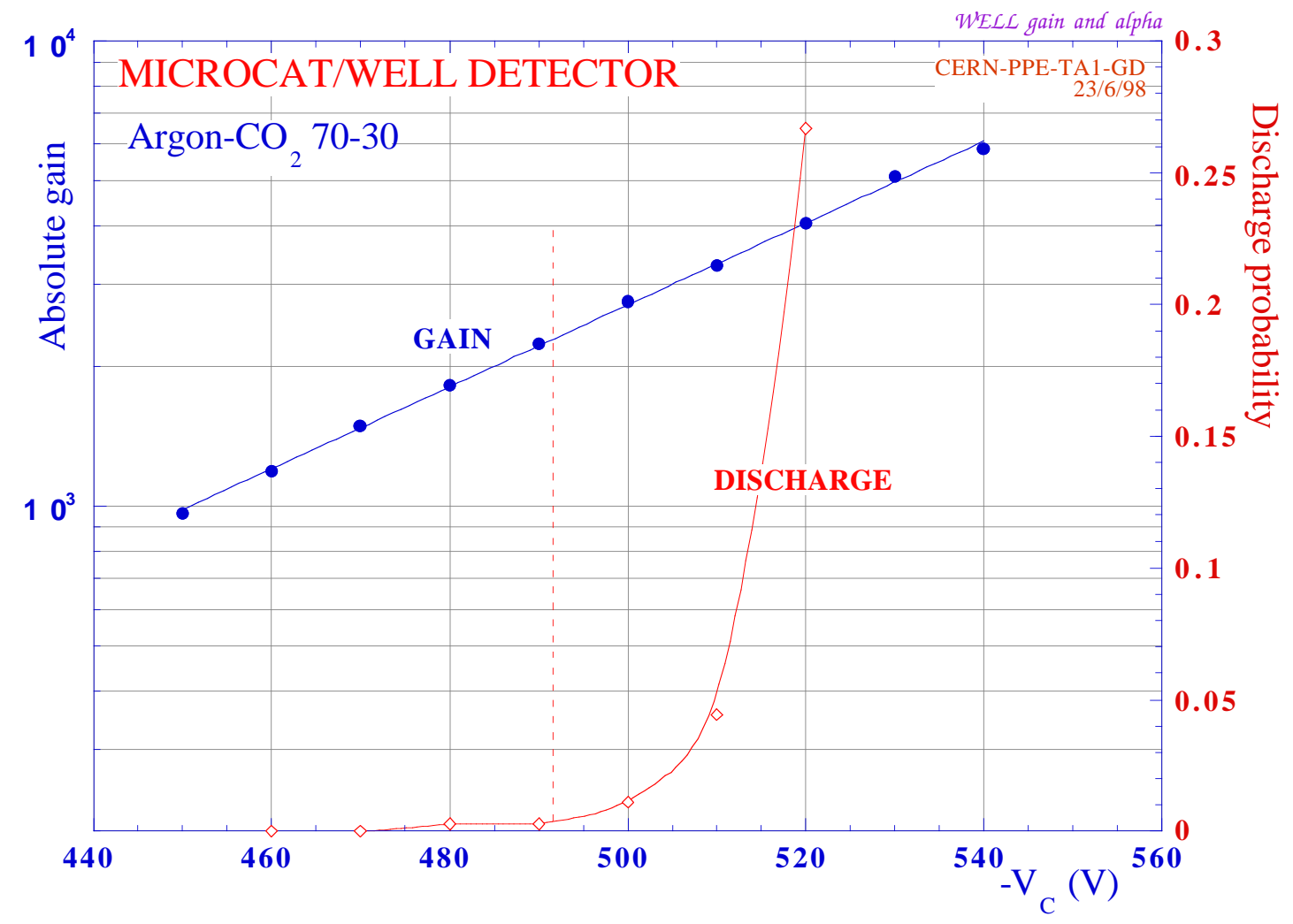

Fig. 12 


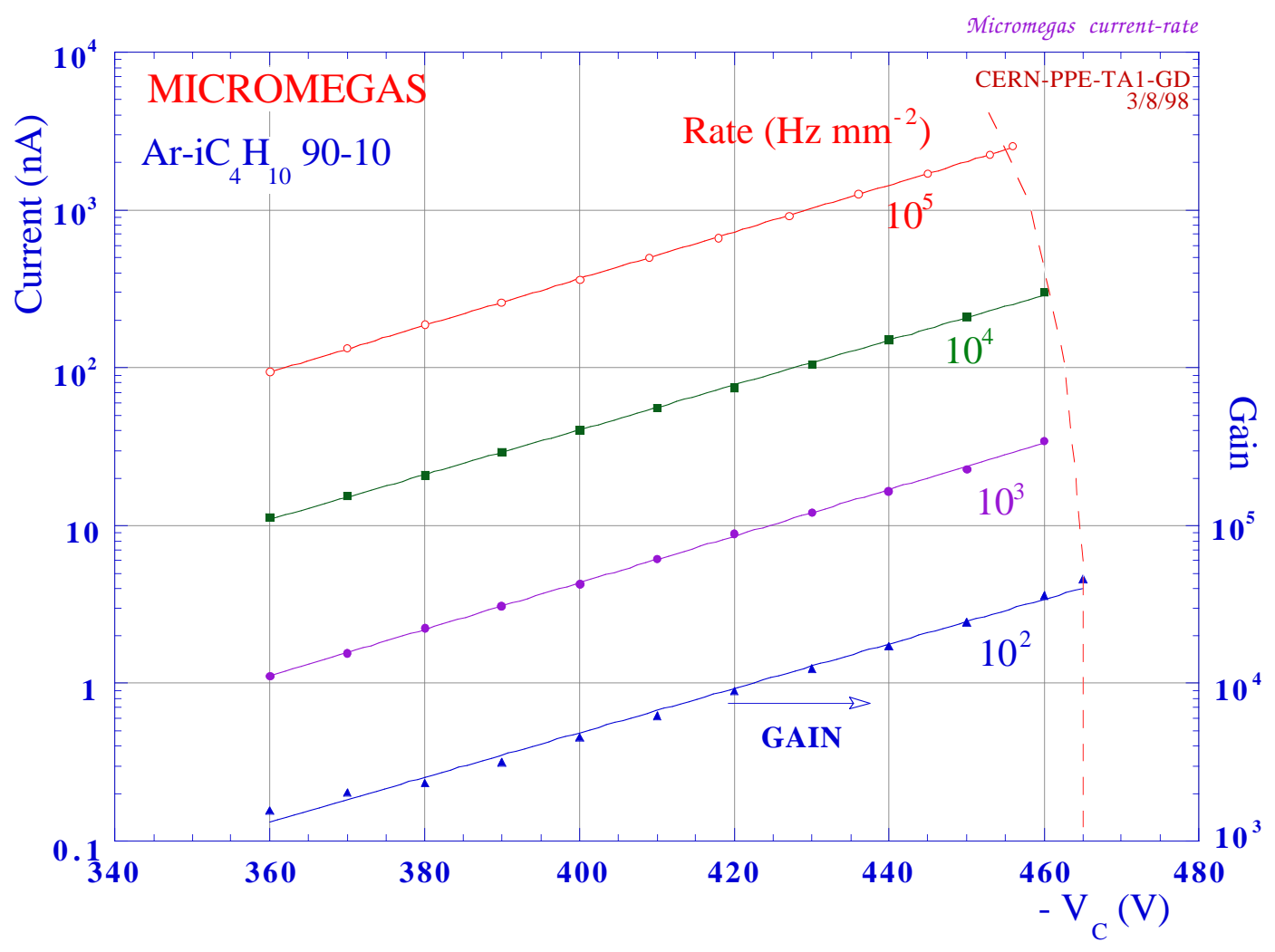

Fig. 13

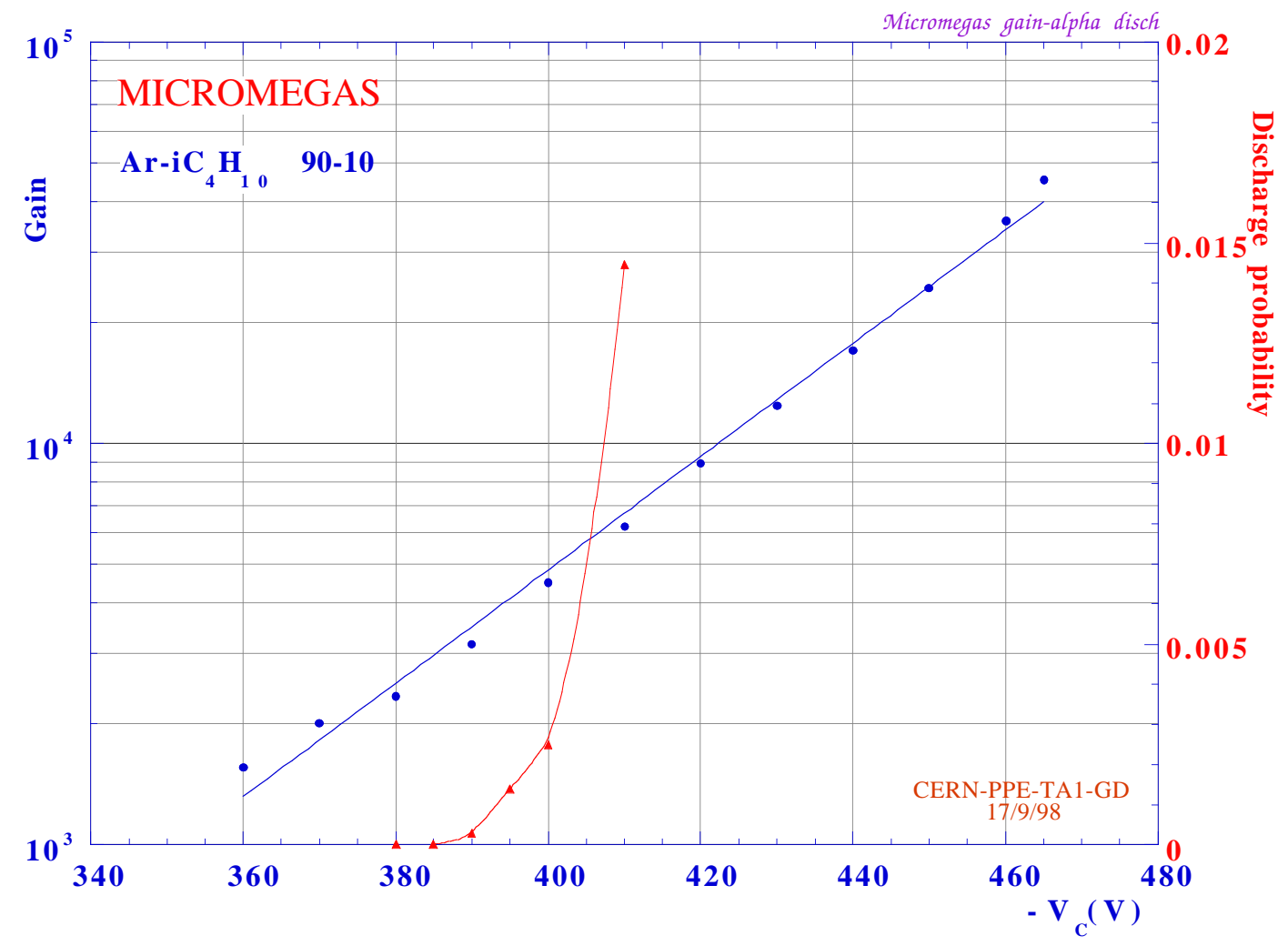

Fig. 14 


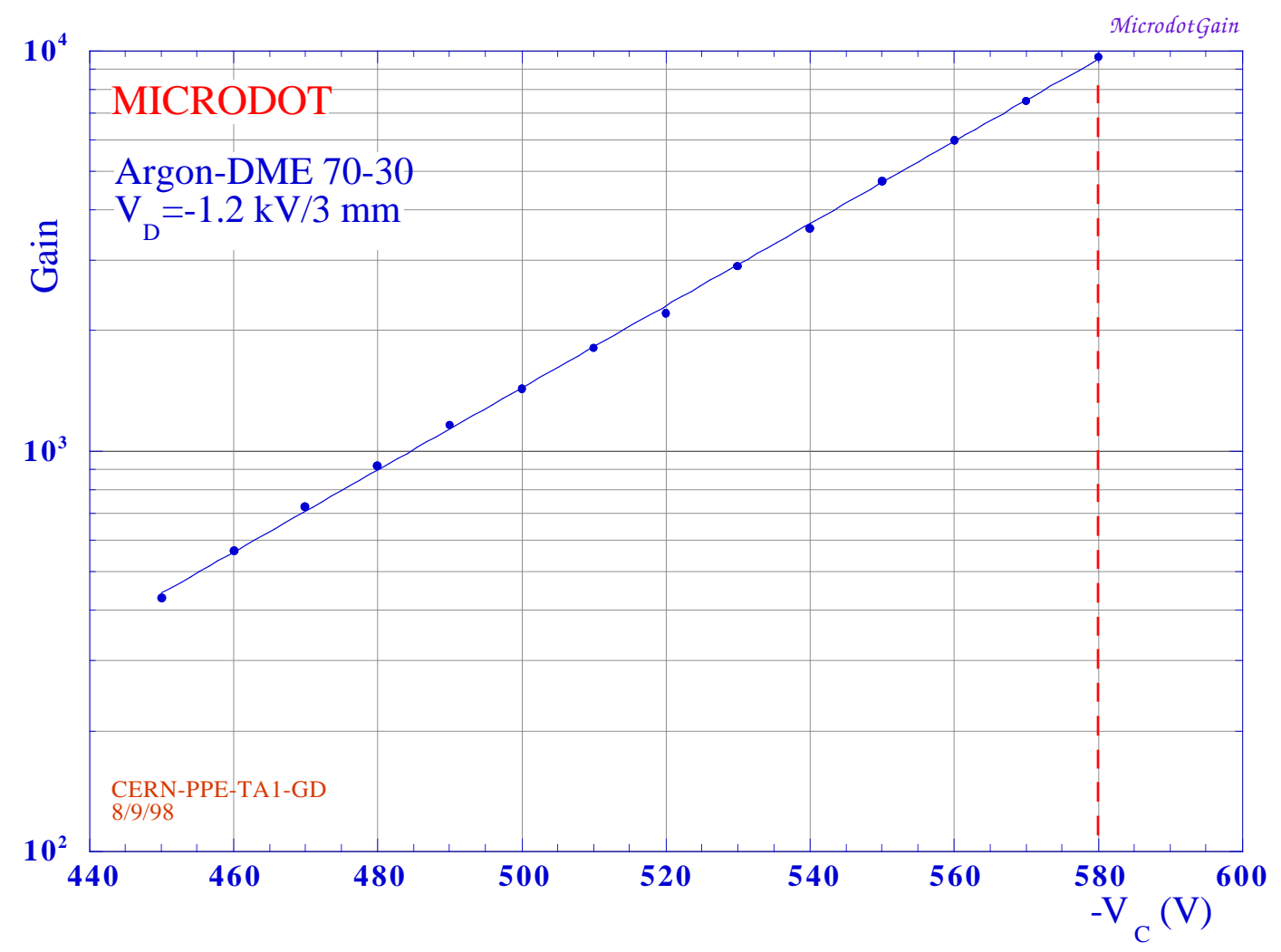

Fig. 15

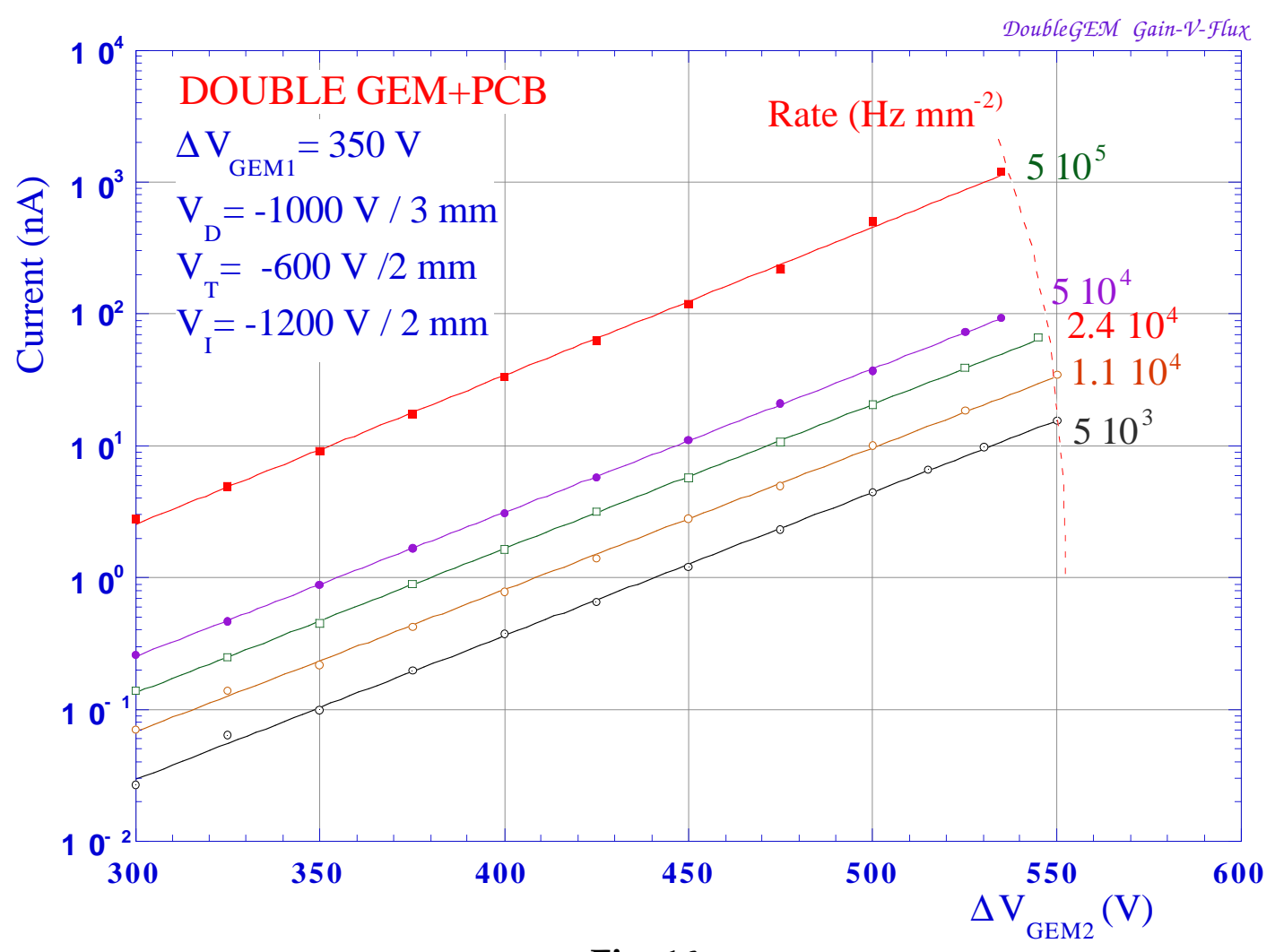

Fig. 16 


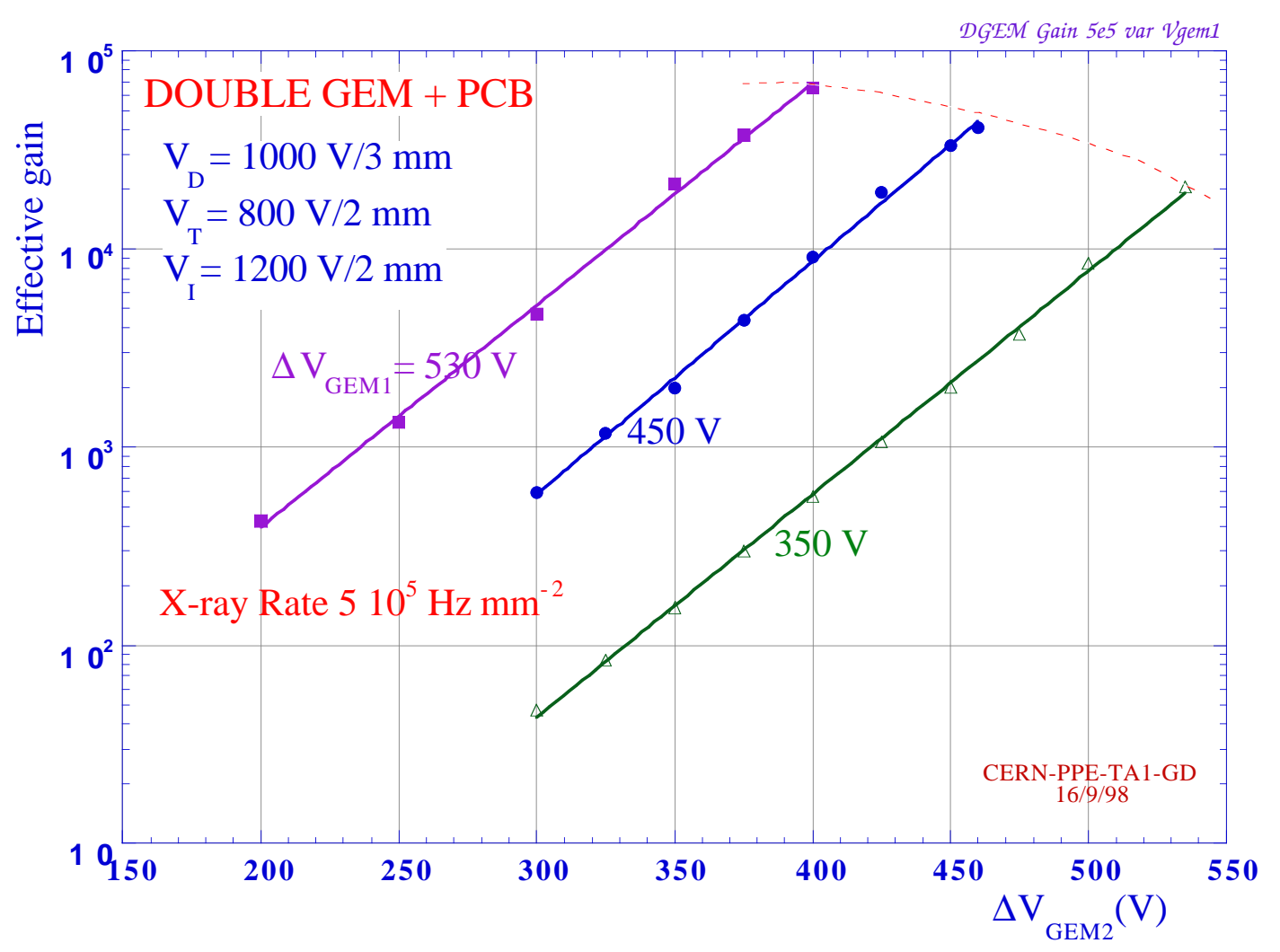

Fig. 17

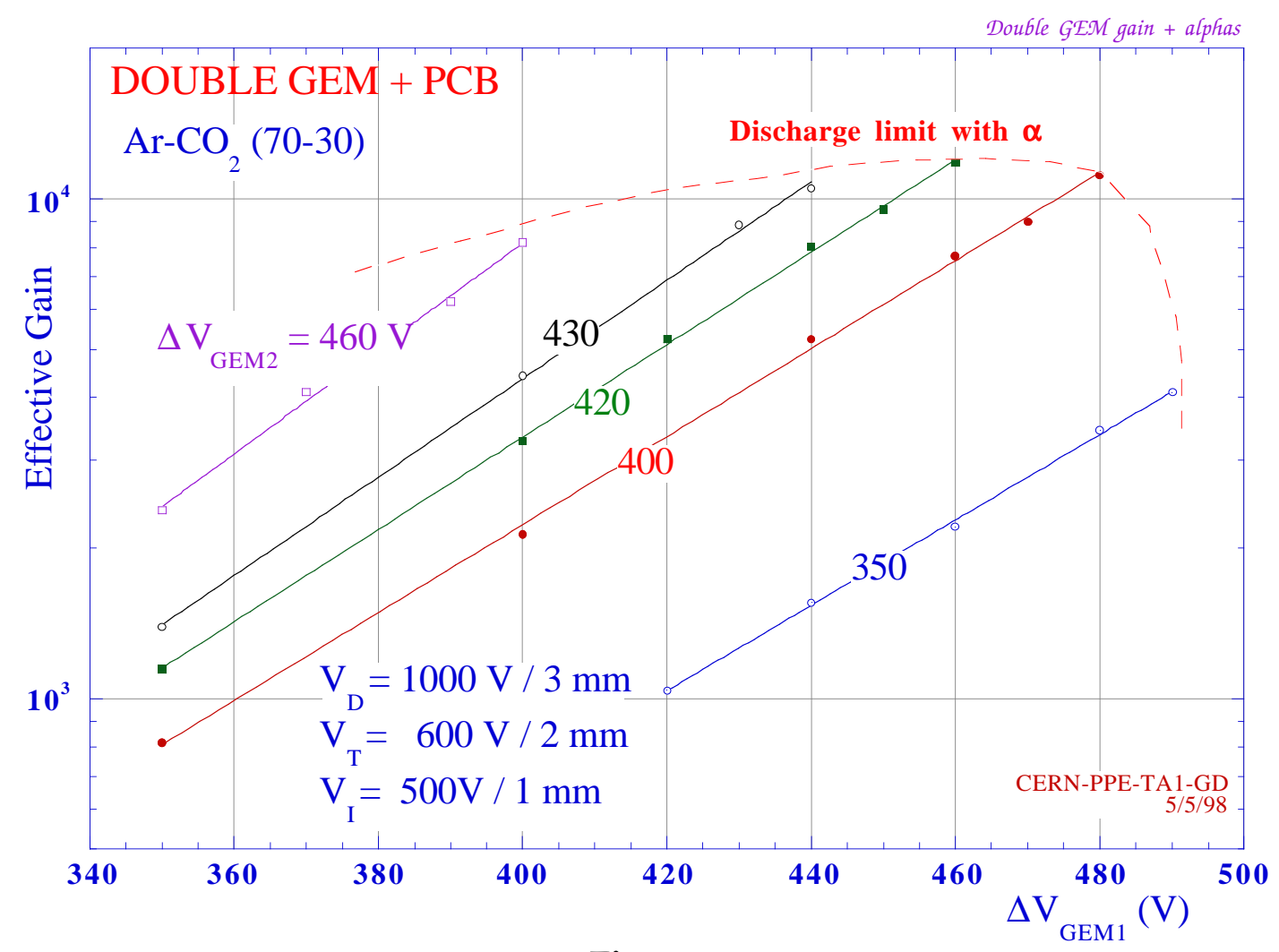

Fig. 18 